\title{
Secondary and Compound Concentrators for Parabolic-Dish Solar-Thermal Power Systems
}

\author{
L. D. Jaffe \\ P. T. Poon
}

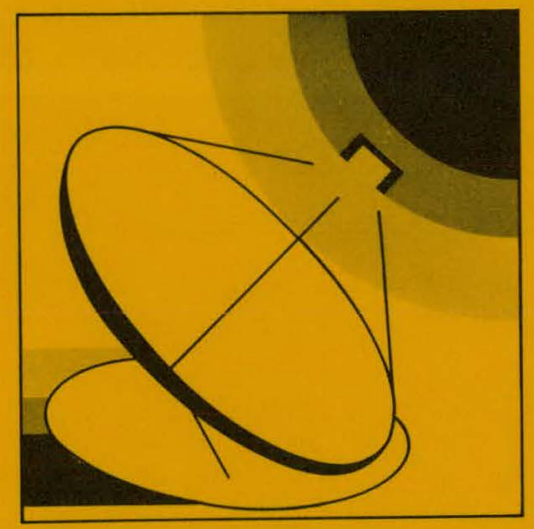

April 15, 1981

Prepared for

U.S. Department of Energy

Through an agreement with

National Aeronautics and Space Administration

by

Jet Propulsion Laboratory

California Institute of Technology

Pasadena, California

(JPL PUBLICATION 81-27) 


\section{DISCLAIMER}

This report was prepared as an account of work sponsored by an agency of the United States Government. Neither the United States Government nor any agency Thereof, nor any of their employees, makes any warranty, express or implied, or assumes any legal liability or responsibility for the accuracy, completeness, or usefulness of any information, apparatus, product, or process disclosed, or represents that its use would not infringe privately owned rights. Reference herein to any specific commercial product, process, or service by trade name, trademark, manufacturer, or otherwise does not necessarily constitute or imply its endorsement, recommendation, or favoring by the United States Government or any agency thereof. The views and opinions of authors expressed herein do not necessarily state or reflect those of the United States Government or any agency thereof. 


\section{DISCLAIMER}

Portions of this document may be illegible in electronic image products. Images are produced from the best available original document. 


\section{Secondary and Compound Concentrators for Parabolic-Dish Solar-Thermal Power Systems}

L. D. Jaffe
P. T. Poon

April 15, 1981

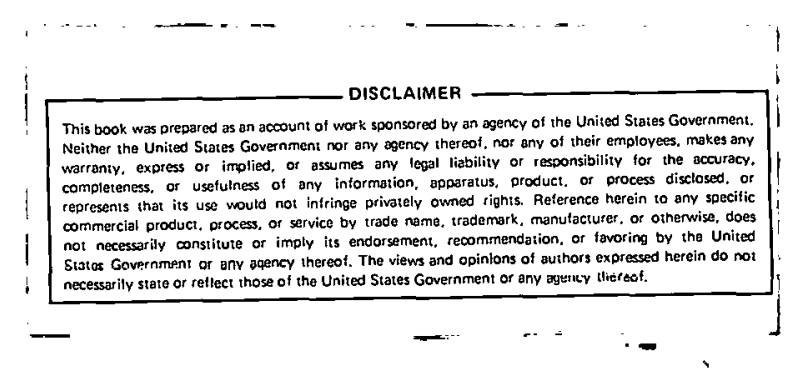

Prepared for

U.S. Departmeril ul Eriergy

Through an agreement with

National Aeronautics and Space Adrririsistration

by

Jet Propulsion Laboratory

California Institute of Technology

Pasadena, California

(JPL PUBLICATIUN 81-27) 
Prepared by the Jet Propulsion Laboratory, California Institute of Technology, for the U.S. Department of Energy through an agreement with the National Aeronautics and Space Administration.

The JPi Solar 1 hermal Power Systems Project is sponsored hy the U.S. Department of Fnergy and forms a part of the Solar Thermal Program to develop low. cost snlar thermal and plertrir pouer plante.

This report was prepared as an account of work sponsored by the United States Government. Neither the United States nor the United States Department of Energy, nor any of their employees, nor any of their cuntractors, subcontracturs, or their employees, makes any warranty, express or implied, or assumes any legal liability or responsibility for the accuracy, completeness or usefulness of any information, apparatus, product or process disclosed, or represents that its use would not infringe privately owned rights. 
A secondary optical element may be added to a parabolic dish solar concentrator to increase the geometric concentration ratio attainable at a given intercept factor. This secondary may be a Fresnel lens or a mirror, such as a compound elliptic concentrator or a hyperbolic trumpet. At a fixed intercept factor, higher overall geometric concentration may be obtainable with a long focal length primary and a suitable secondary matched to it. Use of a secondary to increase the geometric concentration ratio is more likely to be worthwhile if the receiver temperature is high and if errors in the primary are large.

Folding the optical path with a secondary may reduce cost by locating the receiver and power conversion equipment closer to the ground and by el iminating the heavy structure needed to support this equipment at the primary focus. Promising folded-path configurations include the Ritchey-Chrétien and perhaps some three-element geometries. Folding the optical path may be most useful in systems that provide process heat.

\section{ACKNOWLEDGMENT}

The authors thank L. C. Wen, A. Meinel, R. Winston, and P. Shlichta for their comments and suggestions. 
A secondary optical element added to a parabolic dish solar thermal concentrator can increase the geometric concentration ratio attainable at a given intercept factor and can fold the optical path. The increased geometric concentration may be used (1) to reduce receiver losses, (2) to permit higher receiver temperatures and so increase engine efficiency, or (3) to lower concentrator cost by permitting use of a primary of lower optical quality and so of lower cost. The secondary itself should be small and thus can be relatively inexpensive.

A secondary used to increase the concentration ratio may be a lens, such as a Fresnel, or a mirror, such as a compound elliptic concentrator (CEC) or a hyperbolic trumpet. A secondary may be retrofitted to an existing concentrator to upgrade performance. However, adding a secondary to an existing primary is unlikely to give as high an optical performance as can he obtained by designing the compound concentrator as such. One reason is that a higher overall geometric concentration may be obtainable at a fixed intercept factor with a primary of a long focal length and suitable secondary than with a short focus primary and a secondary matched to it. Use of a secondary to increase the geometric concentration ratio is more likely to be worthwhile if the receiver temperature is high and if errors in the primary are large.

Use of a secondary to fold the optical path may reduce cost by permitting shorter ducts to the receiver and power conversion equipment, by locating these components closer to the ground and so reducing maintenance costs, and by eliminating the relatively heavy structure needed to support the receiver and power conversion equipment at the focus of the primary. Promising folded-path configurations include the Ritchey-Chrétien modification of the conventional Cassegrainian and perhaps some three-element geometries. Folding the optical path may be most useful in systems that provide process heat.

A secondary or tertiary element has associated reflection or transmission losses which tend to lower the overall optical efficiency. On the other hand, a suitably designed secondary or tertiary may collect and refocus energy which otherwise would fall outside the receiver aperture, thus increasing the intercept factor over that obtained with the primary alone. This will tend to raise the overall optical efficiency.

In development of compound concentrators for solar thermal systems, areas requiring special attention include optics, heat transfer, materials and system design. Many of these problems center about the materials for the secondary element, the cooling of this element, and possible utilization of the heat deposited in the secondary for preheating the working fluid going to the receiver. Significant work is underway that is applicable to these problems; some of it is centered around solar thermophotovoltaic systems and some around the already devel oped area of laser optics. Additional effort is needed to identify the possibilities and problems of compound concentrators for solar thermal systems and to address the problems identified. 


\section{CONTENTS}

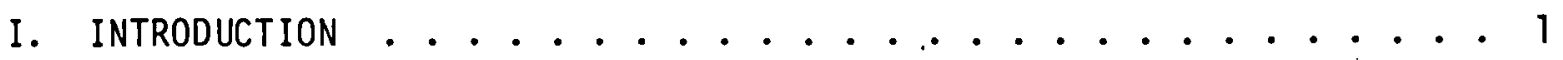

II. OPTICAL PERFORMANCE PARAMETERS OF SOLAR CONCENTRATORS . . . . 7

A. COLLECTION OF NET THERMAL ENERGY BY SOLAR RECEIVER . . . . . 7

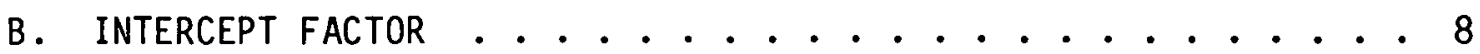

C. GEOMETRIC CONCENTRATION RATIO .............. 9

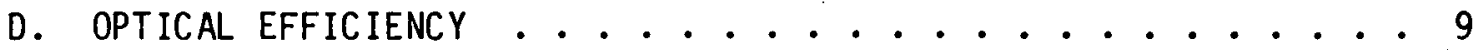

E. FLUX DISTRIBUTION .............. 9

F. EFFECTS OF SECONDARY ON PERFORMANCE .......... 10

III. SeCONDARIES TO INCREASE CONCENTRATION ............ 11

A. CONCENTRATING SECONDARY LENSES . . . . . . . . 12

B. SECONDARY IMAGING AND CONCENTRATING MIRRORS . . . . . 12

C. NON-IMAGING SECONDARY CONCENTRATING MIRRORS . . . . . 12

1. Conical Secondary Mirrors ............ 12

2. Compound Elliptic Secondary Concentrating Mirrors . . . 15

3. Hyperbolic Trumpet Secondary Concentrating Mirrors. . . . 18

4. Other Non-Imaging Secondaries ......... 20

IV. SECONDARIES TO FOLD THE OPTICAL PATH ............ 21

A. CASSEgRAINIAN CONFIGURATIONS . . . . . . . . 22

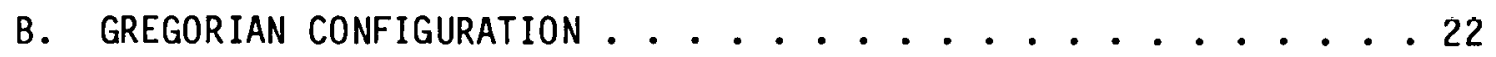

C. RITCHEY-CHRETIEN AND OTHER CORRECTING SECONDARIES . . . . 22

D. THREE-ELEMENT CONCENTRATORS . . . . . . . . 26

v. AREAS NEEDING ATTENTION . . . . . . . . . . 29

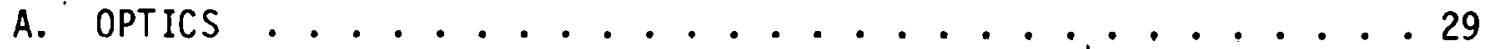

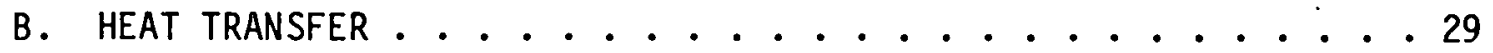




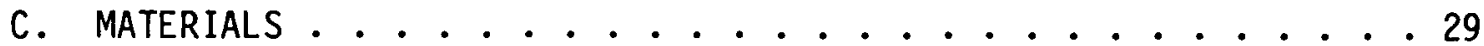

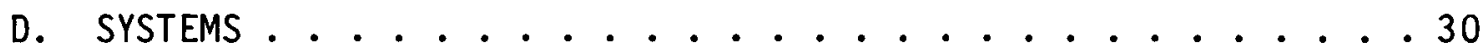

E. PERTINENT WORK ON OTHER APPLICATIONS . . . . . . . 30

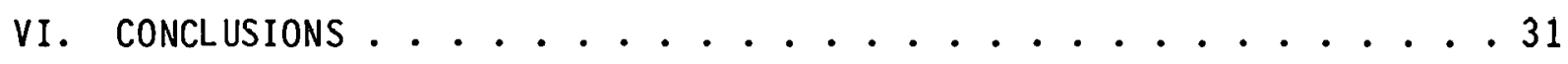

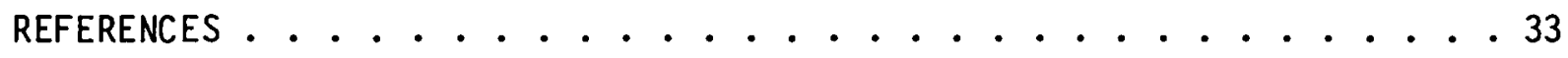

APPENDIXES

A. CURRENT WORK ON SOLAR THERMOPHOTOVOLTAICS ........ 37

B. CURRENT WORK ON LASER OHTILS . . . . . . . . . . . 39 


\section{Figures}

1. Increasing Concentration Ratio with a Secondary Concentrator

(Fresne1 Lens Secondary)................................... 2

2. Folding Optical Path wi th a Secondary Concentrator.................. 3

3. Increasing Concentration Ratio and Folding Optical Path with a

Secondary Imaging Mirror: Schwarzchild Configuration............... 13

4. Increasing Concentration Ratio with a Truncated Conical Secondary

Mirror ("Axicon") ........................................... 14

5. Increasing Concentration Ratio with a Secondary Mirror Consisting

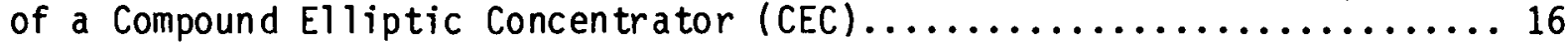

6. Geometric Concentration Ratio versus Focal Ratio of Parabolic Primary Mirror, wi th and wi thout a CEC Secondary.................. 17

7. Increasing Concentration Ratio with a Hyperbolic. Trumpet

Secondary Mirror.......................................... 19

8. Cassegrainian or Ritchey-Chrétien Configurations with Different

Positions of the Secondary.................................. 23

9. Cassegrainian or Ritchey-Chrétien Configuration with Very Short

Focal Length Primary....................................... 24

10. Folding Optical Path with a Secondary Mirror: Gregorian

Configuration........................................... 25

11. Folding Optical Path and Increasing Concentration Ratio - Possible

Three-Element Configuration: Cassegrainian or Ritchey-Chrétien

plus CEC.............................................

12. Folding Optical Path and Increasing Concentration Ratio - Possible

Three-Element Configuration: Short-Focus Ritchey-Chrétien plus

Concave Correcting/Folding Mirror............................. 28

$\underline{\text { Table }}$

1. Compound Point-Focusing Solar Concentrators..................... 4 


\section{SECTION I}

\section{INTRODUCTION}

One approach to production of electricity or high-temperature process heat from solar energy is to use point-focusing, two-axis pointing concentrators in a solar thermal system with a receiver on each concentrator. Concentrators for these systems have traditionally been parabolic mirrors, hence the name, "parabolic dish systems." With the growing emphasis on low cost and high performance, a variety of concepts are being examined to lower the cost of parabolic mirrors and to provide alternatives. Among these alternative concepts are inflated membrane mirrors, faceted and Fresnel mirrors, and Fresnel lenses (Ref. 1). An approach which also warrants consideration is the use of compound concentrators. A compound solar concentrator is a concentrator in which the sunlight is reflected or refracted more than once. It generally consists of a primary mirror or lens, whose aperture determines the amount of sunlight gathered, and a smaller secondary mirror or lens. Additional small optical elements also may be incorporated.

There already is some interest in compound solar concentrators, especially in line-focusing geometries (Refs. 2 through 5). This paper discusses some of the possibilities and problems in using compound concentrators in parabolic dish sys tems.

Configurations for two-element concentrators may be divided into two general classes: those in which the overall optical path is longer than that of the primary alone and those in which it is shorter. A secondary that reduces the overall optical path to less than that of the primary alone can reduce the size of the focal spot and thus increase the geometric concentration, which is often desired (Ref. 6). A secondary which increases the optical path will increase the size of the focal spot and thus have the undesirable effect of lowering the geometric concentration ratio. The chief advantage of this type of secondary is that it permits a small secondary mirror to fold the optical path from a primary mirror to provide a focus at or behind the plane of the primary (as seen from the sun; Ref. 7). This location for the receiver and associated equipment may be more desirable than a position well in front of the primary mirror.

Thus, secondary concentrators in solar thermal dish systems have two principal functions:

(1) Increasing the concentration ratio (Figure 1 )

(2) Folding the optical path (Figure 2)

Advantages and problems of various types of secondaries (Table 1) for each of these purposes will be discussed below.

The cost and the optical losses of the secondary itself must be weighed against the advantages of using a secondary optical element. The secondary should be kept small to minimize its cost. For a small secondary to intercept the light from the primary, it must be located near the primary focus. A small secondary intercepting the radiation concentrated by the primary will be subjected to high heat flux. Therefore, the secondary must be capable of operating at a high temperature or it must be cooled. The problem of heating the secondary will be less severe if optical losses at the secondary are lower. obviously, 


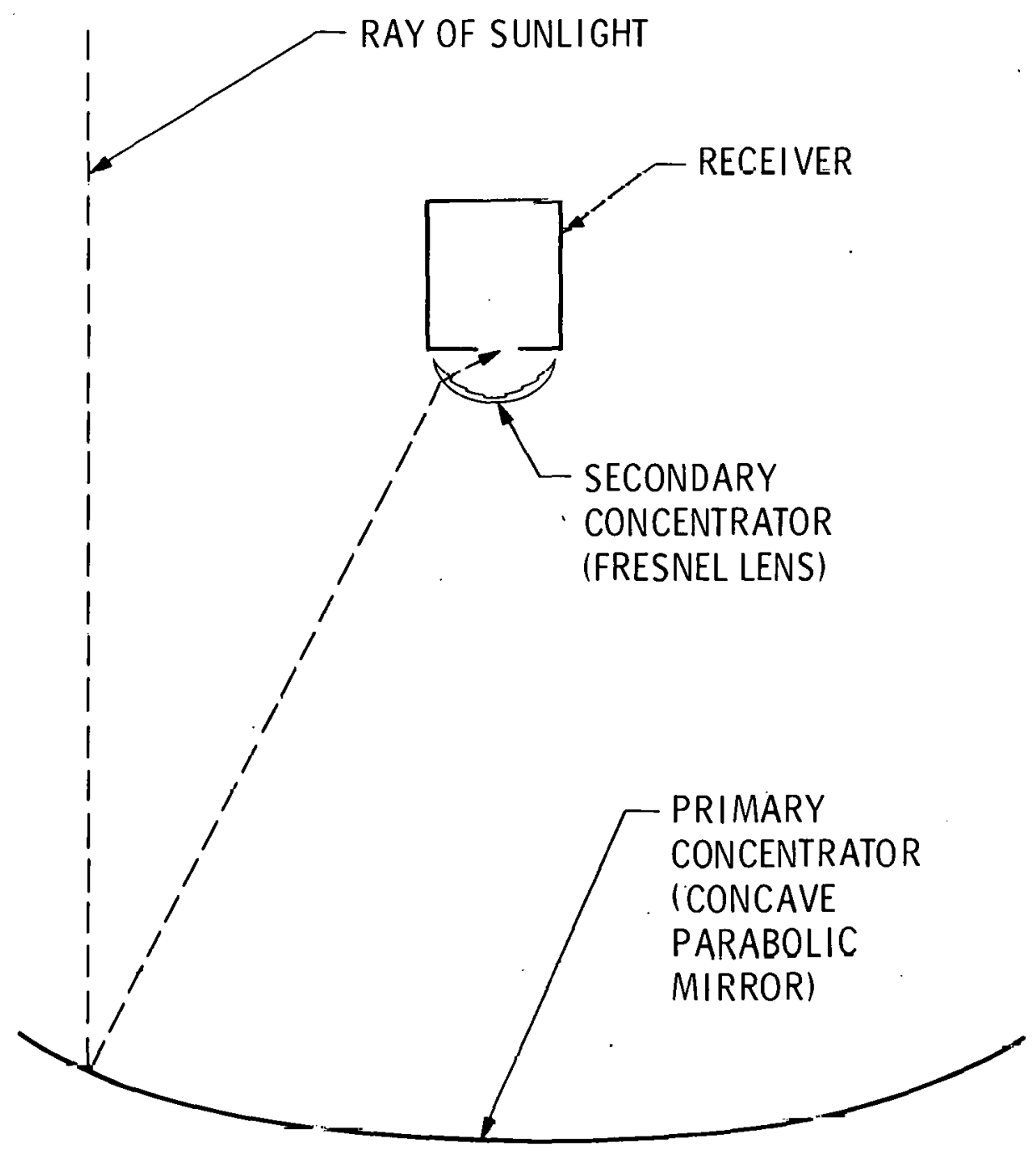

Figure 1. Increasing Concentration Ratio with a Secondary Concentrator (Fresnel Lens Secondary) 


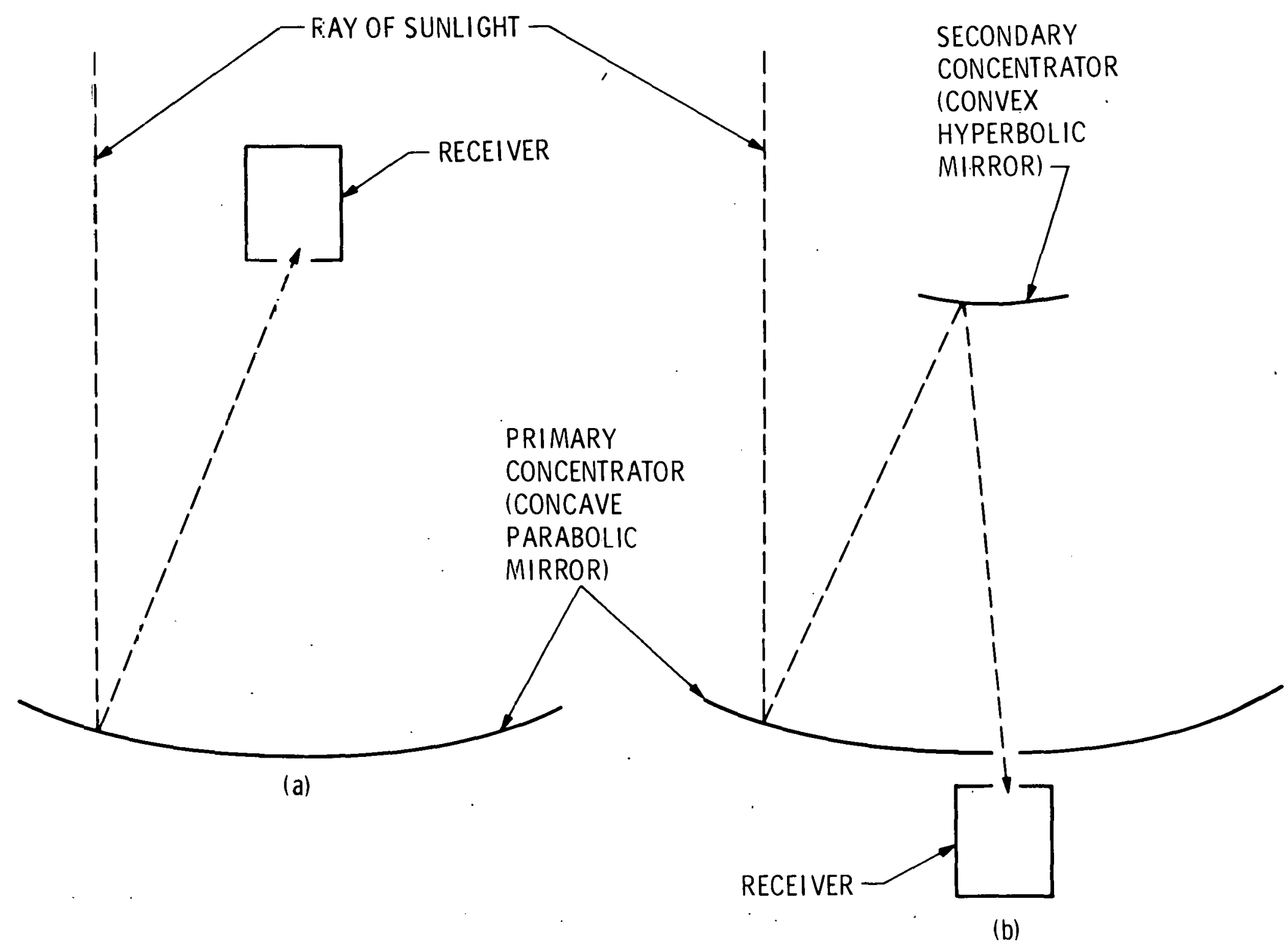

Figure 2. Folding Optical Path with a Secondary Concentrator (Compare (a) conventianal configuration with (b) Cassegrainian configuration, noting position of receiver. Power conversion equipment and ducting may be located with receiver.) 
Table 1. Compound Point-Focusing Solar Concentrators

A. Two-element concentrators

1. Concentrators in which secondary shortens the overall focal length (increases concentration)

a. Secondary element: a lens

(1) Conventional lens

(2) Fresnel lens

b. Secondary el ement: a mirror

(1) Imaging mirror

(a) Schwarzchild configuration

(2) Non-imaging mirror

(a) Conical mirror ("Axicon")

(b) Compound elliptic (CEC) and compound parabolic (CPC) mirrors

(c) Hyperbolic trumpet mirror

2. Concentrators in which the secondary folds the optical path (shortens the physical length)

a. Secondary also decreases the overall focal length

(1) Schwarzchild configuration (see above)

b. Secondary increases the overall focal length

(1) Cassegrainian configuration

(2) Gregorian configuration

(3) Ritchey-Chrétien configuration

B. Configurations wi th three or more elements 
keeping optical losses at the secondary low will also help maintain efficiency. However, the overall optical efficiency is not necessarily lowered by the presence of the secondary -- it may even be increased (by increasing the intercept factor, as discussed later in this paper). 
THIS PAGE

\section{WAS INTENTIONALLY LEFT BLANK}


The optical performance of a solar concentrator depends upon a number of factors including the geometrical configuration of the optics, the optical surface characteristics such as slope errors and specular angular spreading, the pointing error, the reflectance or transmittance, blocking and shadowing, etc. The geometric configuration includes the geometric shape of the elements involved, e.g., spherical, parabolic, elliptic, hyperbolic, as well as the relative locations of the elements. The slope error is a measure of the angular deviation of the actual surface normal of the fabricated concentrator element from that of the ideal geometric surface, and may result from macroroughness due to manufacturing methods, installation misalignment, distortions, and statistical deflections due to wind loads, etc. The specular angular spreading is a measure of the angular spread of the reflected beam from a flat piece of the reflective surface with a well collimated incident beam. The pointing error is the angular offset of the direction of the incoming sunlight from the optical axis of the solar concentrator. When designing secondary or compound solar concentrators, all the above factors must be considered for the individual elements as well as for the optical system as a whole. The resulting optical performance may be specified in terms of the intercept factor, geometric concentration ratio, optical efficiency, and normalized flux distribution.

\section{A. COLLECTION OF NET THERMAL ENERGY BY SOLAR RECEIVER}

Consider a parabolic dish concentrator with an aperture area, A, and reflectance, $\rho$. The net rate of collection of thermal energy, $Q$, by a solar receiver placed at the focus is:

$$
Q=\alpha A \rho I_{0} G \phi(r)-A_{r}\left[\sigma \epsilon\left(T_{r}^{4}-T_{a}^{4}\right)+h\left(T_{r}-T_{a}\right)\right]-Q^{\prime}{ }_{c},
$$

where

$$
\begin{aligned}
\alpha= & \text { effective solar absorptance of the receiver } \\
I_{0}= & \text { incident direct normal insolation } \\
G^{\prime}= & \text { geometric factor to account for shading and blocking due to } \\
& \text { the receiver, supporting structures, etc. } \\
\phi(r)= & \text { intercept factor as a function of the receiver aperture } \\
& \text { radius }=r \text { The intercept factor is the ratio of energy } \\
& \text { entering the receiver aperture to the total concentrated } \\
& \text { energy reaching the focal plane. } \\
A_{r}= & \text { receiver aperture area }=\pi r^{2} \text { for a circular aperture of } \\
\sigma= & \text { Stefius = } r \text {. } \\
\epsilon= & \text { effective emissivity of the solar receiver } \\
T_{r}= & \text { receiver temperature (absolute) } \\
T_{a}= & \text { ambient temperature (absolute) } \\
h^{\prime}= & \text { total convective heat transfer coefficient, which includes the } \\
& \text { temperature-dependent natural convective heat transfer coeffi- } \\
& \text { cient and the forced convective heat transfer coefficient, } \\
& \text { which depends upon wind velocity } \\
Q^{\prime} c= & \text { rate of loss of energy by conduction }
\end{aligned}
$$


The geometric concentration ratio, $C$, is defined as

$$
C=\frac{A}{A_{r}}=\frac{A}{\pi r^{2}}
$$

The expression for $Q$ may be formulated in terms of $C$ as follows

$$
Q=A\left\{\alpha \rho I_{0} G \phi(A / C)-(1 / C)\left[\sigma \epsilon\left(T_{r}{ }^{4}-T_{a}{ }^{4}\right)+h\left(T_{r}-T_{a}\right)\right]\right\}-Q^{\prime}{ }_{c},
$$

where the dependence of the intercept factor on the receiver aperture radius has been replaced by the dependence on $A / C$.

The above expression may be generalized to include a secondary concentrator. As an example, for a two-element concentrator with reflective surfaces characterlzed by reflectance values, $\mathrm{N}_{1}, \mathrm{~N}_{2}$, the rate of collection nf net thermal energy by a solar receiver placed at the focal region of the two-element optical system is given by

$$
Q=A\left\{\alpha \rho_{1} \rho_{2}{ }^{n} I_{0} G_{12} \phi_{12}(A / C)-(1 / C)\left[\sigma \epsilon\left(T_{r}{ }^{4}-T_{a}{ }^{4}\right)+h\left(T_{r}-T_{a}\right)\right]\right\}-Q^{\prime}{ }_{c},
$$

Here the exponent, $n$, is the average number of reflections at the secondary. The geometric factor, $G_{12}$, includes the shading and blocking effects of the additional optical element, and the intercept factor, $\rho_{12}$, is determined by optical analysis of the two-element concentrator.

The above equation shows a trade-off between the geometric concentration ratio, $C$, and the intercept factor, $\phi_{12}$. Suppose the value of $C$ is increased and all the other factors are fixed. The first term will be decreased because $\phi 12$ Increases monotonically with $A / C$. However, the magnitude of the seconduterm is al so decreased because of its inverse dependence upon $C$. Thus, the performance equation yields a value for $Q$ (the net rate of collection of thermal energy) which will be maximum for some value of $C$. In a realistic optimization process, other factors such as the flux distribution must be taken into account since the receiver temperature, $T_{r}$, which governs the heat losses, depends upon the solar flux distribution impinging upon the receiver surfaces.

The optical efficiency, which will be discussed below, is

and

$$
\eta_{0}=\rho G \phi \quad \text { with a simple concentrator }
$$

$$
n_{0}=\rho_{1} \rho_{2}^{n_{G}}{ }_{12} \phi_{12} \text { wi th a two-element concentrator }
$$

\section{B. INTERCEPT FACTOR}

The intercept factor, $\phi$, is the ratio of the energy entering a receiver aperture of a given size to the total concentrated energy reaching the focal plane. It is an important quantity because the rate at which solar energy enters the receiver is proportional to the intercept factor. To be meaningful, the intercept factor for a given solar concentrator must be specified together with the receiver aperture size or, equivalently, the geometric concentration ratio. Usually intercept factors of 0.95 or higher are of interest. 


\section{GEOMETRIC CONCENTRATION RATIO}

For solar thermal power the overall concentration of the whole beam is of importance rather than the peak concentration at the center of the whole beam. The overall concentration can be expressed as the geometric concentration ratio, $C$, which is the ratio of the concentrator aperture area to the receiver aperture area, for a given intercept factor. It is important to state the intercept factor when specifying the geometric concentration ratio. For example, a pinhole receiver aperture placed at the focus of any solar concentrator may result in an enormous geometric concentration ratio, but the intercept factor would be extremely small. Stating geometric concentration ratio without specifying the intercept factor gives no information on how well the solar concentrator performs.

When considering the concentrator alone wi th the receiver not defined, the receiver aperture, and hence the geometric concentration ratio, may be treated as an adjustable parameter. With a given concentrator, there is (as indicated above) a trade-off between intercept factor and geometric concentration ratio. If the intercept factor is low, much of the concentrated solar energy will not enter the receiver aperture and will be lost. If the intercept factor is higher with this same concentrator, the geometric concentration ratio will be lower, the receiver aperture will be larger, and hence the re-radiation losses from the receiver will be larger.

The geometric concentration needed to provide reasonable receiver performance increases with the receiver temperature. At high temperatures, where re-radiation becomes very important, a small receiver aperture (high geometric concentration ratio) is highly desirable. Typical geometric concentration ratios for solar thermal dish systems are 200 to 5000 at an intercept factor of 0.95 or higher.

\section{OPTICAL EFFICIENCY}

The optical efficiency, $n_{0}$, is the ratio: (energy delivered to the receiver aperture by the concentrator)/(solar energy incident upon the primary concentrator). For a single mirror, this efficiency depends upon its reflectance; for a pair of mirrors, on the product of their reflectances; for a lens, on its transmittance (which is influenced by the absorption within the lens and the reflection losses at its surfaces). Shadowing and blocking by objects in the optical path, surch as structural el ements, receiver, etc., as well as the intercept factor, contribute to losses in optical efficiency.

\section{E. FLUX DISTRIBUTION}

The normalized fiux distribution is the flux distribution nedr the focus of the solar concentrator expressed as a ratio to the incident solar flux. For a fixed receiver aperture size, the focal plane flux distributions from two different solar concentrators may have the same intercept factor, the same geometric concentration ratio, and carry the same amount of energy, but may differ in the way in which the flux is distributed within the receiver aperture. Knowledge of the normalized flux distribution is essential in the design of a receiver to match the concentrator since it affects the temperature distribution and the thermal stresses on the receiver. 


\section{F. EFFECTS OF SECONDARY ON PERFORMANCE}

As stated in the Introduction, there are two types of secondary concentrators: those that increase the geometric concentration ratio for a fixed intercept factor (or, equivalently, that increase the intercept factor for a fixed receiver aperture size) and those used to fold the optical path so that the receiver can be placed in a more desirable location. The first type will permit a smaller receiver aperture size for the delivery of the same amount of energy into the receiver and consequently will have reduced re-radiative and convective losses. However, there will be additional reflective or transmissive losses because of the presence of the secondary element, and the trade-off may result in a higher or lower optical efficiency, depending upon the optical design. Introducing a secondary to fold the optical path does not reduce re-radiative and convective losses; therefore, optical efficiency is usually lowered by the introduction of such a secondary.

The optimum geometric concentration attainable for a given recciver temperature with a simple concentrator (a single optical element) depends principally upon the focal ratio (focal length/diameter) and the slope errors of the opticial surface. A secondary can increase the optimum concentration ratio because no practical primary concentrator is ideal. The primary cannot attain the thermodynamically possible solar concentration ratio of 46,000 because of optical aberrations. A parabolic primary mirror, for example, has a maximum geometric concentration ratio of about 11,000 at an intercept factor of 1.0 . 
An increase in geometric concentration means that the solar radiation can be passed through a smaller receiver aperture, and hence re-radiation and other aperture losses from the receiver can be reduced. This reduction in thermal losses may be used to increase the collector efficiency at constant receiver temperature, or to increase the receiver temperature at constant collector. efficiency. An increase in receiver temperature may be desirable either to satisfy a demand for process heat at a higher temperature or to permit operation of a heat engine at higher Carnot efficiency and therefore at higher actual efficiency. Thus, a secondary concentrator which increases the geometric concentration may allow the system efficiency to increase or provide heat at a higher temperature, or both.

For a maximum concentration ratio, light should leave the last optical element and enter the receiver aperture over as large a solid angle as possible. The maximum possible solid angle from the axis is $2 \pi$ for a planar receiver aperture such as that of a cavity receiver. If the sunlight reaches the receiver aperture over a more limited angular range, the attainable concentration ratio (at a given intercept factor) will be lower. Thus, a secondary concentrator may be used to provide a high input solid angle to the receiver, while allowing constraints upon the primary to be relaxed. For example, use of a secondary may allow the primary concentrator and the receiver to be farther apart.

The increased geometric concentration provided by a secondary concentrator may also be used to lower concentrator cost. If a primary concentrator (without secondary) is to provide high concentration, it must have low optical errors and high optical accuracy. Therefore, it will probably cost more than a concentrator of lower quality. By adding a secondary concentrator, it may be possible to achieve the same overall concentration ratio with a primary of 1 ower quality and cost. Since the small secondary should be relatively inexpensive, the total concentrator cost may be reduced.

In general, the higher the receiver temperature and the larger the errors in the primary, the more likely it is that use of a compound concentrator to increase the geometric concentration ratio will be worthwhile.

As previously mentioned, a secondary concentrator should be small to minimize cost. If it is to increase concentration, it must reduce the focal length of the system and thus must itself have a very short focal length. Since in a two-element concentrator the receiver is located at the focus of the secondary, this type of secondary concentrator must be located close to the receiver. It may therefore be advantageous to mount the secondary concentrator on the receiver or to design it as an integral part of the receiver. A secondary concentrator element located close to the receiver is called a "terminal concentrator." -(See References 8 and 9.)

A secondary optical element for increasing the geometric concentration ratio can be either a lens or a mirror. If a mirror is used, it may be either of the conventional imaging type or of the non-imaging type. These alternatives are discussed below. 
The possibility of using a secondary lens in a concentrator for solar thermal power seems to have received very little consideration. It probably has been assumed that the cost of such a lens would be prohibitive. This assumption is not necessarily true.

For example, if the diameter of the primary is 10 meters and its geometric concentration ratio is 1000 , the diameter of a terminal secondary would be about $30 \mathrm{~cm}$. The desired secondary concentration probably will be between 1.5 and 10. For such a low secondary concentration, a very poor lens should be adequate. Absorption and consequential heating in the lens are likely to prove troublesome, as di scussed below. Fresnel lenses (Figure 1) are thinner than conventional lenses of the same diameter and may be desirable in order to reduce absorption and heating. Because both primary concentrator and receiver will subtend large angles as seen from the secondary, the light will cross the lens surfaces with a wide angular spread. This characteristic may make it difficult to design Fresnel facets wi th edges that do not intercept an appreciable fraction of the light and refract it away from the receiver. This problem apparently has not been investigated for solar secondary concentrators. Another problem is that it is difficult to make single-element lenses with the very short focal ratios desired; the practical limit is about 0.7 .

To reduce losses, an anti-reflection surface treatment or coating is desirable and probably cost effective. With such a treatment the reflection losses might be less than $5 \%$.

No work, apparently, has been done on the design of secondary lenses for solar thermal power systems, nor any analysis of the optical performance of a compound concentrator using a secondary lens and suitable for solar thermal applications.

\section{B. SECONDARY IMAGING AND CONCENTRATING MIRRORS}

A secondary mirror placed close to the focus of the primary concentrator can be used to increase overall concentration. A conventional concave mirror could provide a small image of the sun (high concentration) if the focal length of this secondary is short.

With this geometry (called a Schwarzchild configuration), the secondary mirror folds the optical path. The receiver and the power conversion subsystem (if one is used) are between the primary concentrator and the secondary, nearer the latter (Figure 3 ). If the secondary is small, the receiver and power conversion equipment will block a considerable portion of the beam reaching the secondary from the primary. Accordingly, this geometry is of little practical interest.

\section{NON-IMAGING SECONDARY CONCENTRATING MIRRORS}

\section{Conical Secondary Mirrors}

A variety of secondary mirrors can be used which do not fold the optical path and can provide concentration without the blockage problem mentioned above. These are mirrors which do not form an image. Perhaps the simplest is the truncated conical mirror, often called the "Axicon" (Figure 4). The light from the primary 


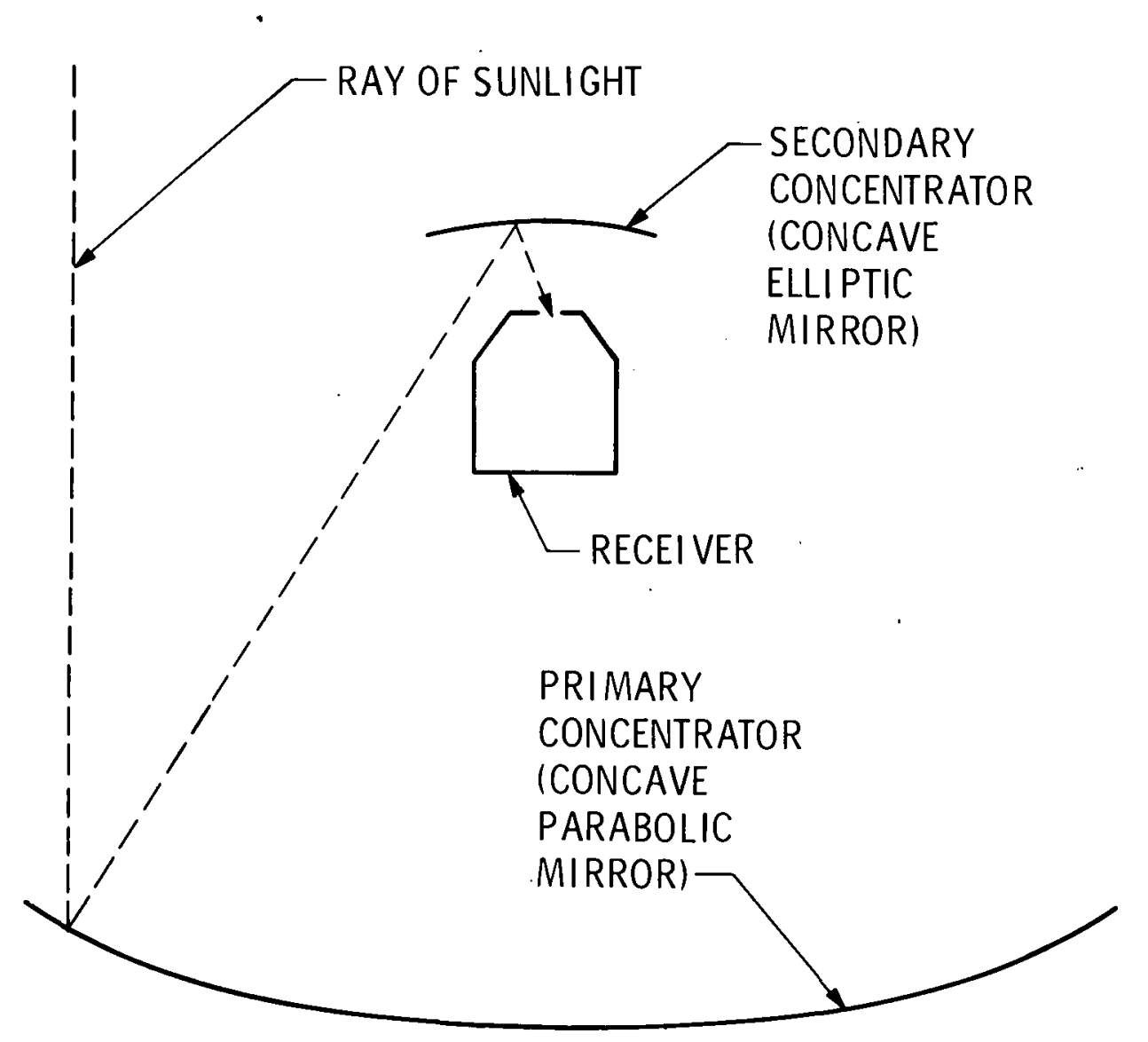

Figure 3. Increasing Concentration Ratio and Folding Optical Path with a Secondary Imaging Mirror: Schwarzchild Configuration (Note that receiver tends to block optical path from primary to secondary.) 


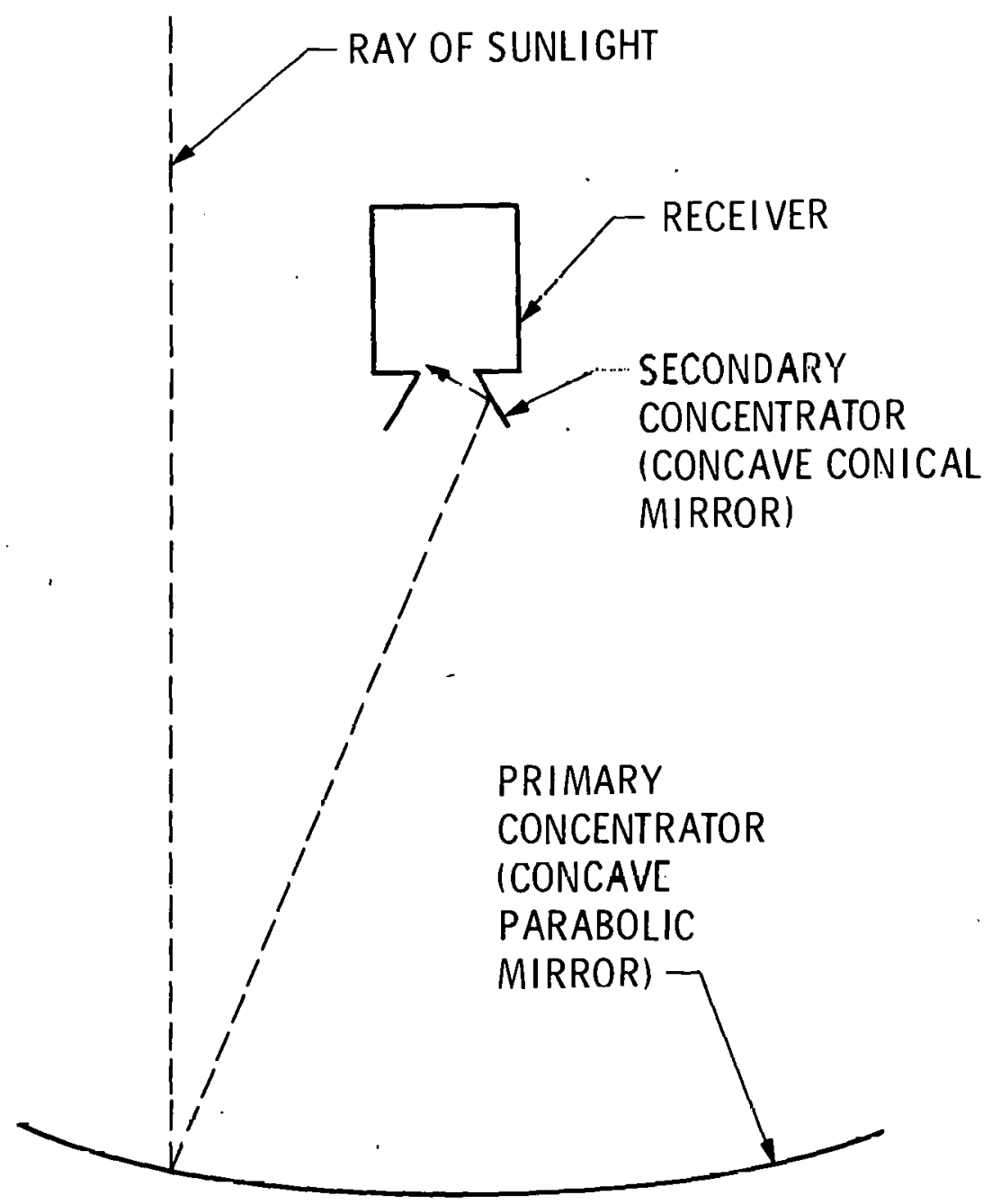

Figure 4. Increasing Concentration Ratio with a Truncated Conical Secondary Mirror ("Axicon") 
enters the large end of the cone, wi th the receiver aperture at the small end. Some analysis of the performance of this optical geometry has been carried out (Refs. 8 and 10). The results indicate that the performance will not be as good as that of other non-imaging secondaries discussed below. However, because of its simple shape, the Axicon is probably easier to manufacture, more readily mass-producible, and may be cost-effective.

\section{Compound Elliptic Secondary Concentrating Mirrors}

The compound parabolic concentrator (CPC; called a "parabolotor ic focone" in the Russian literature) has attracted considerable attention as a primary solar concentrator, especially for line-focusing geometries (Refs. 10 through 13). The CPC is designed to concentrate incoming light from a distant source such as direct sunlight, rather than the incoming rays from a nearby primary, which a secondary concentrator must handle (Refs. 10 and 14). A modification of the CPC which is more suitable for use as a secondary is the compound elliptic concentrator (CEC) (Refs. 14 and 15). For point focusing, the CEC consists of a toroid whose inner surface is a mirror. In axial section, each half of the mirror is an ellipse and has its focus at the opposite mirror surface (Figure 5 ). One end of the CEC is close to the focal plane of the primary; the receiver aperture is at the other end. Baranov (Ref. 16), Winston and Welford (Ref. 15), Poon and Higgins (Ref. 17), and others (Refs. 2 and 9) have analyzed the optics of compound concentrators using CEC and CPC secondaries. Their work provides an indication of the concentration attainable with parabolic primaries of various focal ratios and various surface slope errors.* The results indicate (to a first approximation) that the geometric concentration provided by the CEC secondary is independent of the surface slope errors of the primary. The secondary provides a fixed geometric concentration which, when multiplied by the primary geometric concentration, gives the overall geometric concentration of the system. For example, depending upon its slope error, an $f / 1.0$ primary may have a geometric concentration ratio of 400 or 1600 at an intercept factor of 0.99 . The secondary can provide an additional ideal concentration of about 4.8 , making the overall geometric concentration ratio for the two cases about 2000 and 8000 , respectively for the same intercept factor.

On the other hand, the geometric concentration attainable by a secondary varies strongly with the focal ratio of the primary. With a given slope error, the geometric concentration attainable by a parabolic primary mirror is maximum at a focal ratio of 0.5 to 0.6 . At this primary focal ratio, a suitable secondary can increase the concentration by a factor of 2 . If the primary focal ratio is 1.0 , a secondary can increase the concentration ratio by almost a factor of 5. At a primary focal ratio of 2.0, a secondary can increase concentration 16 times. The geometric concentration ratio attainable by a compound concentrator therefore can increase as the primary focal ratio increases (Figure 6 ), whereas the concentration ratio attainable by the primary alone decreases (beyond a focal ratio of 0.6$)$. Thus, wi th concentrating secondaries there may be an advantage in using a long-focus primary. The concentration provided by a short-focus primary can be somewhat increased by adding a secondary, but the

\footnotetext{
₹ The surface slope error is a major contributor to spreading of the focal spot beyond its area for a perfect concentrator. Other optical errors, such as imperfect specularity of the mirror, can conveniently be considered equivalent to additional slope error of the primary mirror or lens.
} 


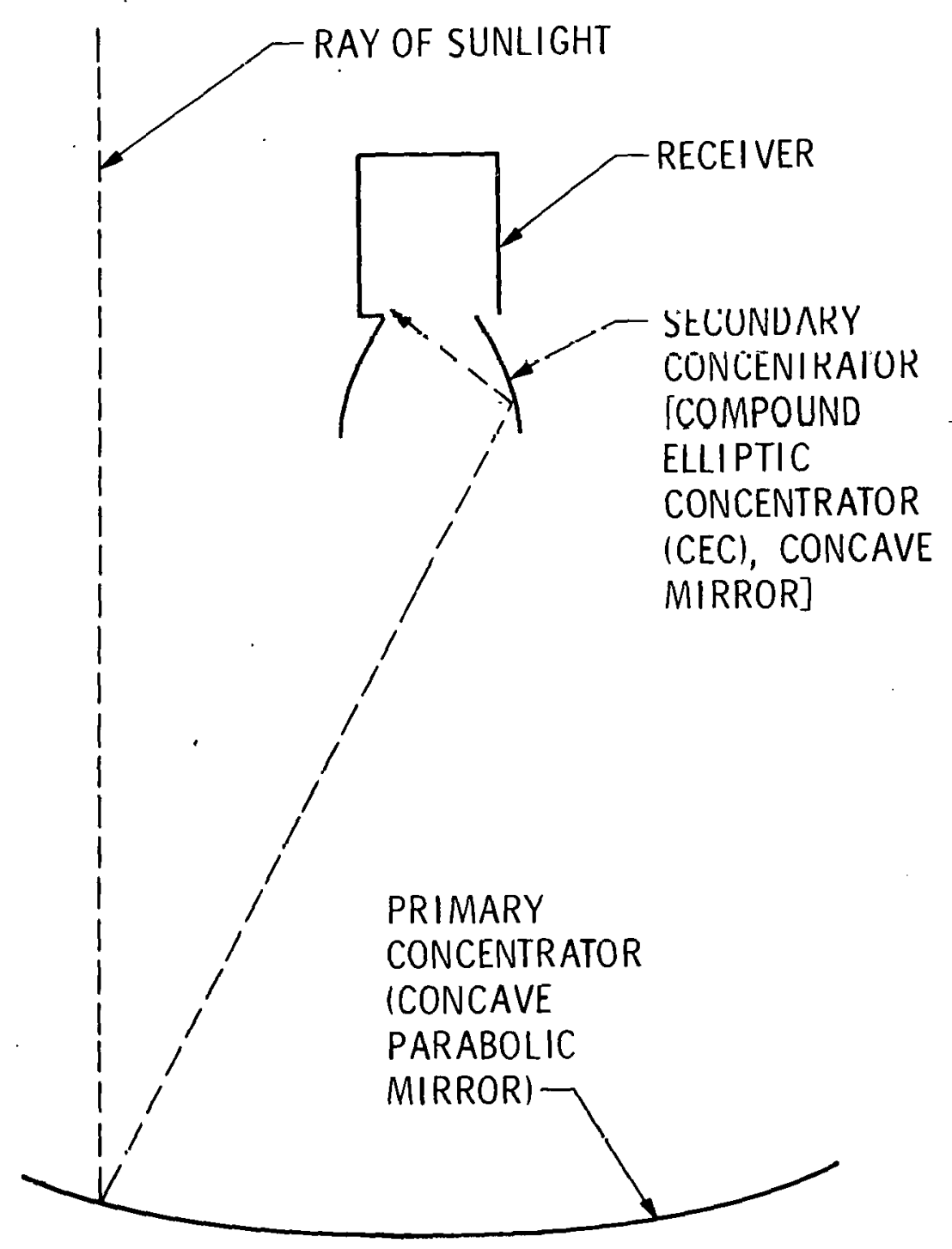

Figure 5. Increasing Concentration Ratio with a Secondary Mirror Consisting of a Compound Elliptic Concentrator (CEC) 


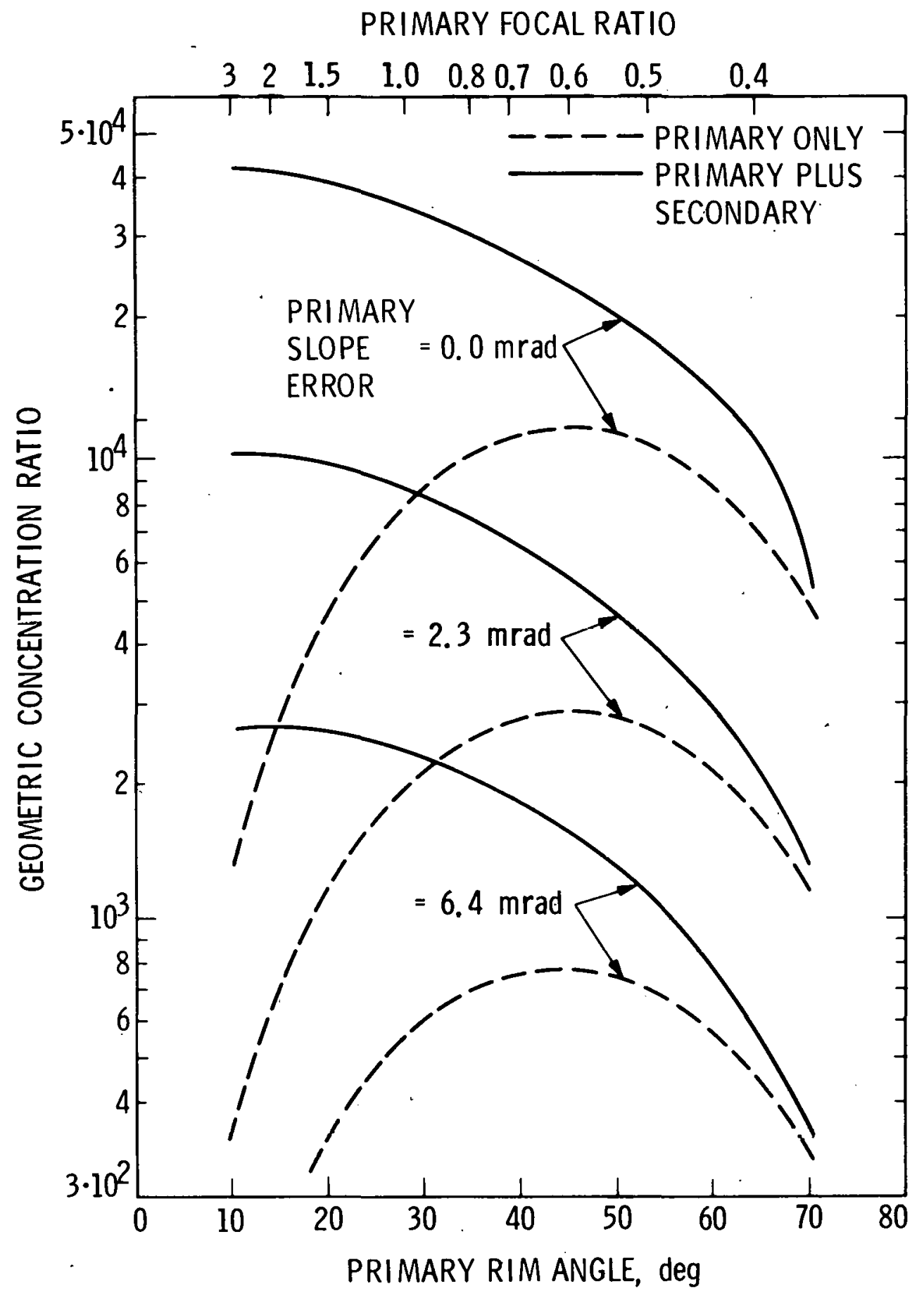

Figure 6. Geometric Concentration Ratio versus Focal Ratio of Parabolic Primary Mirror, with and wi thout a CEC Secondary (Rectangular distribution of slope errors; intercept factor 1.0. Adapted from Baranov, Ref. 16.) 
overall concentration may still be less than that attainable by redesigning for use of a long-focus primary with a suitable secondary. There may be a penalty in structural weight and cost in going to a longer primary focal length, and a trade-off analysis should be done. One means of mitigating the disadvantage of long focal length is folding the optical path as discussed below.

An example of the performance attainable with a primary having moderate slope errors follows: A primary slope error of $4.8 \mathrm{mrad}$ at $\mathrm{f} / 1.0$ permits a primary geometric concentration ratio of 390 . The addition of a CEC secondary permits a further concentration of about 4.5 for an overall concentration of about 1800 (Ref. 18). This suggests the possibility of permitting moderate primary slope errors to reduce the cost of the primary and using a secondary to provide a rather high overall concentration. * The reflection loss at the secondary must also be considered; the trade-off in collector performance is discussed below. The effect of slope errors in the secondary has not been analyzed; it should be small because the secondary itself has a very low concentration ratio. Only rays entering the secondary close to the limits of both the acceptance angle and the secondary entrance aperture should be lost through secondary slope errors.

\section{Hyperbolic Trumpet Secondary Concentrating Mirrors}

Winston and Welford have proposed another geometry for non-imaging secondary concentrating mirrors (Ref. 19). This is a hyperbolic "trumpet" (Figure 7). Its small end is in the primary focal plane and its large end is between the primary and the prime focus. The hyperbolic trumpet may be regarded as a more sophisticated modification of the conical secondary, having a higher performance.

Limited analysis of secondary trumpet performance has been reported (Ref. 20). One example is a parabolic primary mirror with a focal ratio of 0.6 , a slope error of $1.8 \mathrm{mr}$ ad, and a concentration ratio of 2000 at an intercept factor of 1.0 (Ref. 15). The hyperbolic secondary could provide a concentration ratio of about 1.5, resulting in an overall concentration ratio of about 3000 (Ref. 20). Alternatively, the secondary could be used to allow relaxation of the slope tolerance for the primary.

Winston and Welford (Ref. 21) note that the hyperbolic "trumpet" secondary should be less sensitive to alignment error and other sources of beam spread than a CEC secondary and does not increase the overall optical length (unlike the CEC). Another advantage of the hyperbolic trumpet is that it should facilitate increasing the intercept factor: It can gather and refocus light on the aperture that would otherwise be lost, possibly resulting in a significant improvement in optical efficiency. Further, only a small fraction of the light from the primary strikes the secondary (Ref. 18) which reduces the reflection loss at the secondary. One example (Ref. 20), for a mirror with a rectangular distribution of slope errors, shows a higher energy throughput at low and moderate slope errors for a hyperbolic trumpet than for a CEC at the same geometric concentration ratio; the reverse appears to be true with high slope errors. Meinel (Ref. 22) points out that reflections in the CEC tend to occur near grazing angles, at which the reflectance

\footnotetext{
\# Because the secondary is small, its cost should be relatively low. For a $10-m$ primary, the CEC mentioned above has an entrance diameter of $0.5 \mathrm{~m}$ (fixed by the primary focal spot) and a length of $0.7 \mathrm{~m}$.
} 


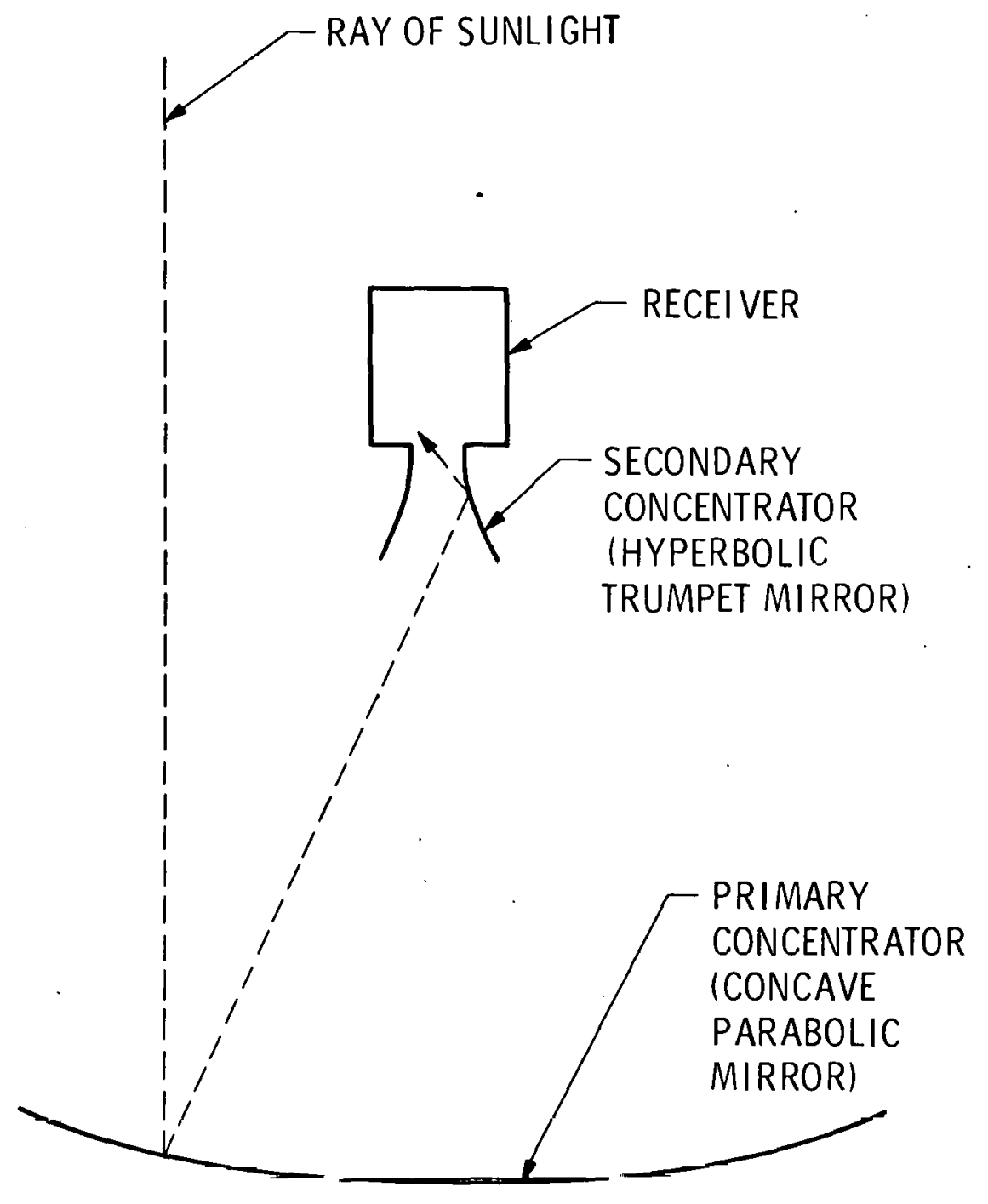

Figure 7. Increasing Concentration Ratio with a Hyperbolic Trumpet Secondary Mirror 
may be exceptionally high; whereas in the hyperbolic trumpet the angles of incidence are near normal. CECs will al so generally be smaller than the corresponding trumpets and therefore may be cheaper. A hyperbolic secondary will shadow more of the primary than a CEC with a similar concentration ratio; this shadowing will increase with the focal length of the primary.

A secondary to upgrade the performance of an existing parabolic dish concentrator at the JPL Parabolic Dish Test Site (Edwards, California) is now being prepared for test.

\section{Other Non-Imaging Secondaries}

Unconventional primary concentrators may be combined with other secondaries. For example, Buzin (Ref. 23) has described a compound concentrator consisting of a conical mirror primary wi th a secondary which is a convex, roughiy conical surface of revolution based on an off-axis parabola. 
Use of a secondary mirror to fold the optical path may lead to cost savings. With the conventional parabolic dish, the receiver and, in many systems, the power conversion subsystem (engine and alternator) are located near the focus, well out on the sunward side of the concentrator and high off the ground. Since these components are heavy, a heavy and therefore somewhat costly structure is needed to support them. If this structure is attached to the reflector structure, its weight tends to deform the latter and hence distort the mirror and degrade its performance. Also, ducting for working fluid, cooling water, and electrical leads may have to be run to and from the receiver and power converter. There may be significant losses associated with these runs. Folding the optical path with a secondary mirror placed sunward (forward) of the primary mirror (Figure 2b) permits placement of the receiver and the power conversion subsystem immediately behind the primary. Since the secondary mirror can be much lighter than the receiver, engine, and alternator, a lighter and cheaper structure can be used to support $i t$, and the loads due to the heavy components can be transmitted more readily to the support without distorting the primary mirror. Ducts to and from the receiver and power conversion subsystem are shortened. Also, the receiver and power conversion subsystem are located closer to the ground, making maintenance easier and cheaper. (Some of these advantages of folding the optical path do not apply if the primary is a lens rather than a mirror.)

Other folding geometries may be considered. A secondary could be used, for example, to turn the optical axis $90^{\circ}$ and bring the beam out to a receiver, thereby reducing blockage of the primary mirror by the receiver and power conversion subsystem. This is possible since the folding mirror, if located near the primary focus, could be smaller in diameter than these other components. Essentially, the same result could be attained by using a similar small mirror near the focus to fold the beam $180^{\circ}$ to a receiver at or behind the primary mirror. The reduction in blockage must also be traded off against the reflection loss at the secondary and the possible structural complications of the off-axis configuration.

A secondary which folds the optical path introduces reflection or transmission loss at the secondary. Also, it usually lengthens the optical path, which tends to reduce the geometric concentration ratio at a given intercept factor. This increases the receiver aperture size and, hence, increases the receiver thermal losses. Optical efficiency therefore often is lowered by a secondary which folds the optical path. However, as discussed below, it is possible to use the folding secondary (or additional optical elements) to reduce optical aberrations, which can permit the geometric concentration ratio to increase and so increase the efficiency, or at least reduce the loss in efficiency which would otherwise occur.

A rather cursory look indicates that folding the optical path may be most useful in systems that provide process heat: shortening the ducts that carry high-temperature fluid to and from the receiver reduces thermal and fluid-dynamic losses; placing the receiver lower should reduce maintenance costs appreciably in such applications. 


\section{A. CASSEGRAINIAN CONFIGURATIONS}

A Cassegrainian optical system utilizes a primary concave parabolic mirror and a secondary convex hyperbolic mirror, located between the primary and the prime focus (Figure $2 b$ ). It is a convenient means of folding the optical path to a receiver at or immediately behind the primary.

To keep the secondary smal1, it must be located near the focus of the primary. The overall optical path is then almost doubled by insertion of the secondary. This configuration almost doubles the diameter of the focal spot and reduces the geometric concentration by almost a factor of four. This design is therefore unlikely to be cost-effective except in systems operating at low receiver temperatures.

The secondary can be placed nearer the primary if it is somewhat larger. If the position of the secondary focus is not changed, the overall optical path will be less than wi th a small secondary (Figure 8), and the loss in geometric concentration also will be less. The secondary, however, will block more of the incoming light to the primary. A hole can be left in the center of the primary corresponding to the area shaded by the secondary, hut. the structure to support the primary will cost more than the structure for a primary of the same reflective area without a hole. The loss in geometric concentration will generally be more serious than the shadowing by the secondary.

In addition to folding the beam, Cassegrainian geometry offers the possibility of using a parabolic primary with a very short focal ratio (shorter than $0.6)$, which gives the maximum geometric concentration on a flat aperture for a paraboloid al one (Ref. 24). The secondary converts the highly convergent primary beam configuration to a less convergent beam that is more suitable to a plane aperture (Figure 9). In this configuration the extremely short-focus primary can provide a higher geometric concentration that may compensate, at least in part, for the decrease normally introduced by the hyperbolic secondary. Such geometries need further investigation. One disadvantage of this design is that the outer portion of the primary is at a high angle to the plane normal to the sun line, and therefore tends to increase mirror area and cost faster than it adds projected area for intercepting and collecting sunlight.

See References 24 through 34 for other analyses of the design of Cassegrainian solar concentrators.

\section{B. GREGORIAN CONFIGURATION}

A Gregorian configuration consists of a concave parabolic primary mirror and a concave elliptic secondary mirror located beyond the primary focus (Figure 10). A Gregorian has a longer overall length than the corresponding Cassegrainian, requires additional structure, and appears to offer no advantage over a Cassegrainian.

\section{RITCHEY-CHRÉTIEN AND OTHER CORRECTING SECONDARIES}

A parabolic primary mirror, if optically perfect, focuses to a point (within the limits of geometrical optics) the light incoming parallel to its optical axis. Light incoming at an angle to the axis is not focused to a point. This optical aberration, known as coma, seriously degrades the concentration attainable with short focus paraboloids for an object such as the sun, which subtends about half a degree. The degradation becomes even worse if the sun is off the optical axis. 


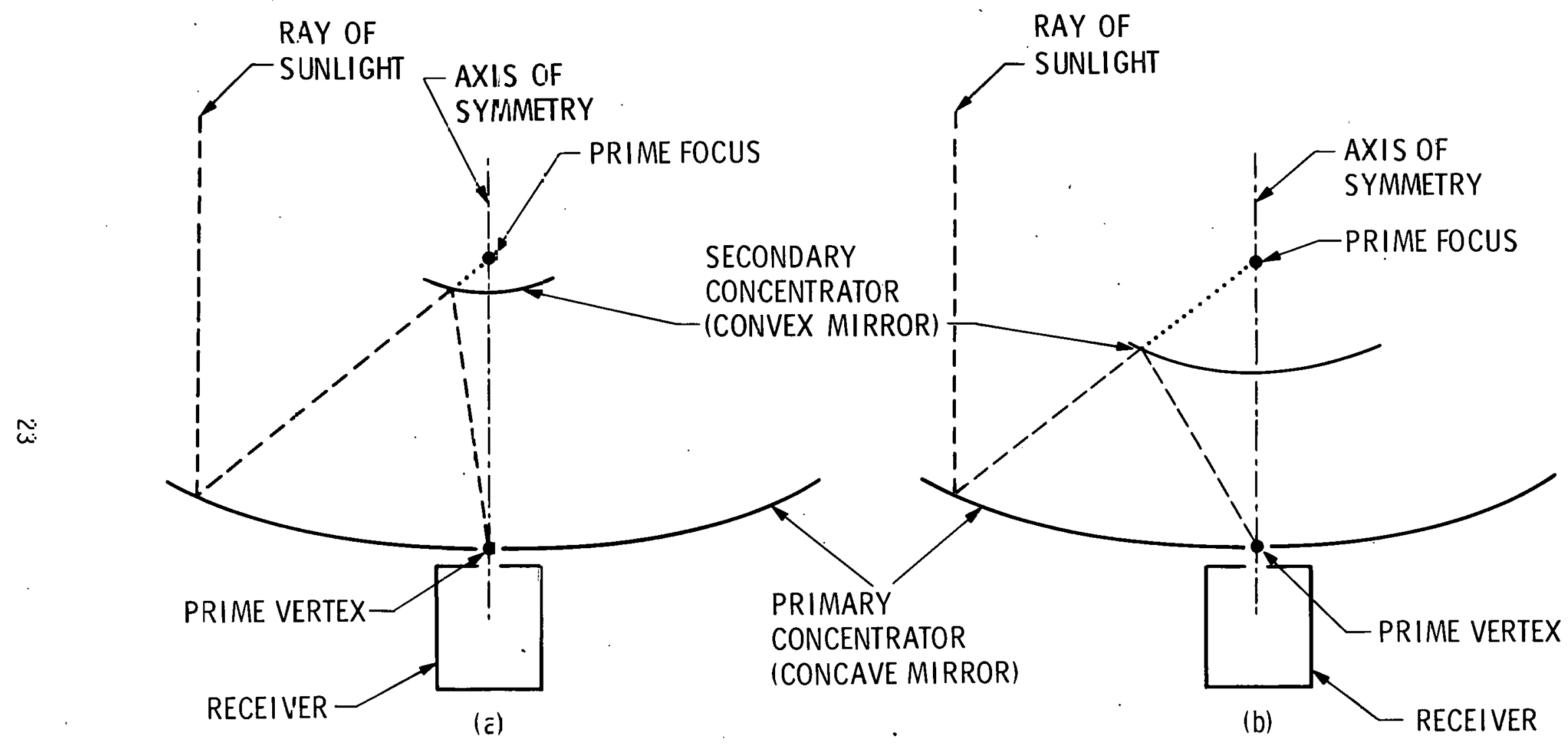

Figure 8. Cassegrainian or Ritchey-Chretien Configurations with Different Pasitions of the Secondary: (a) Secondary near prime focus; (b) Secondary $1 / 3$ way from prime focus to prime vertex: The optical path is shorter in (b). 


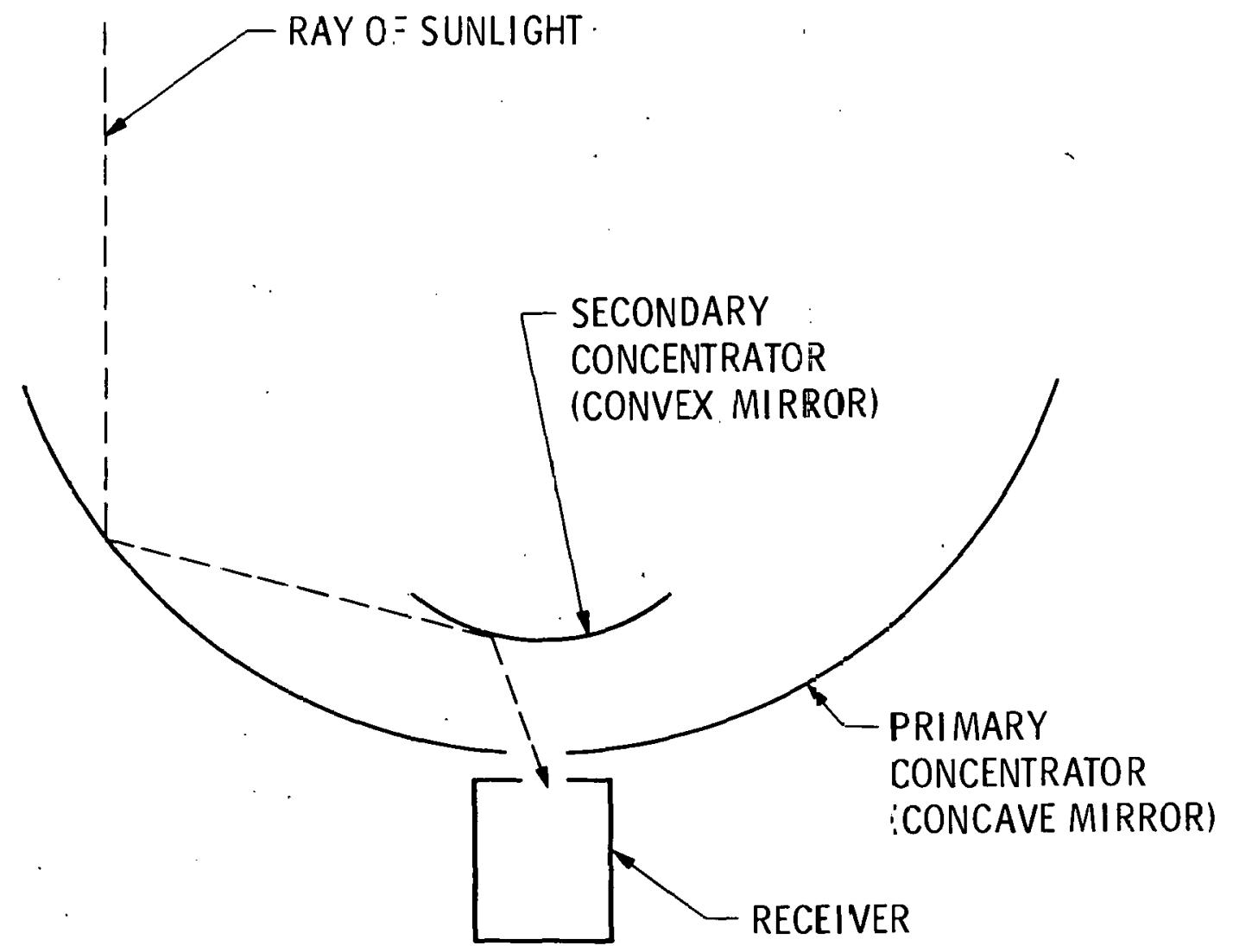

Figure 9. Cassegrainian or Ritchey-Chrétien Configuration with Very Short Focal Length Primary 


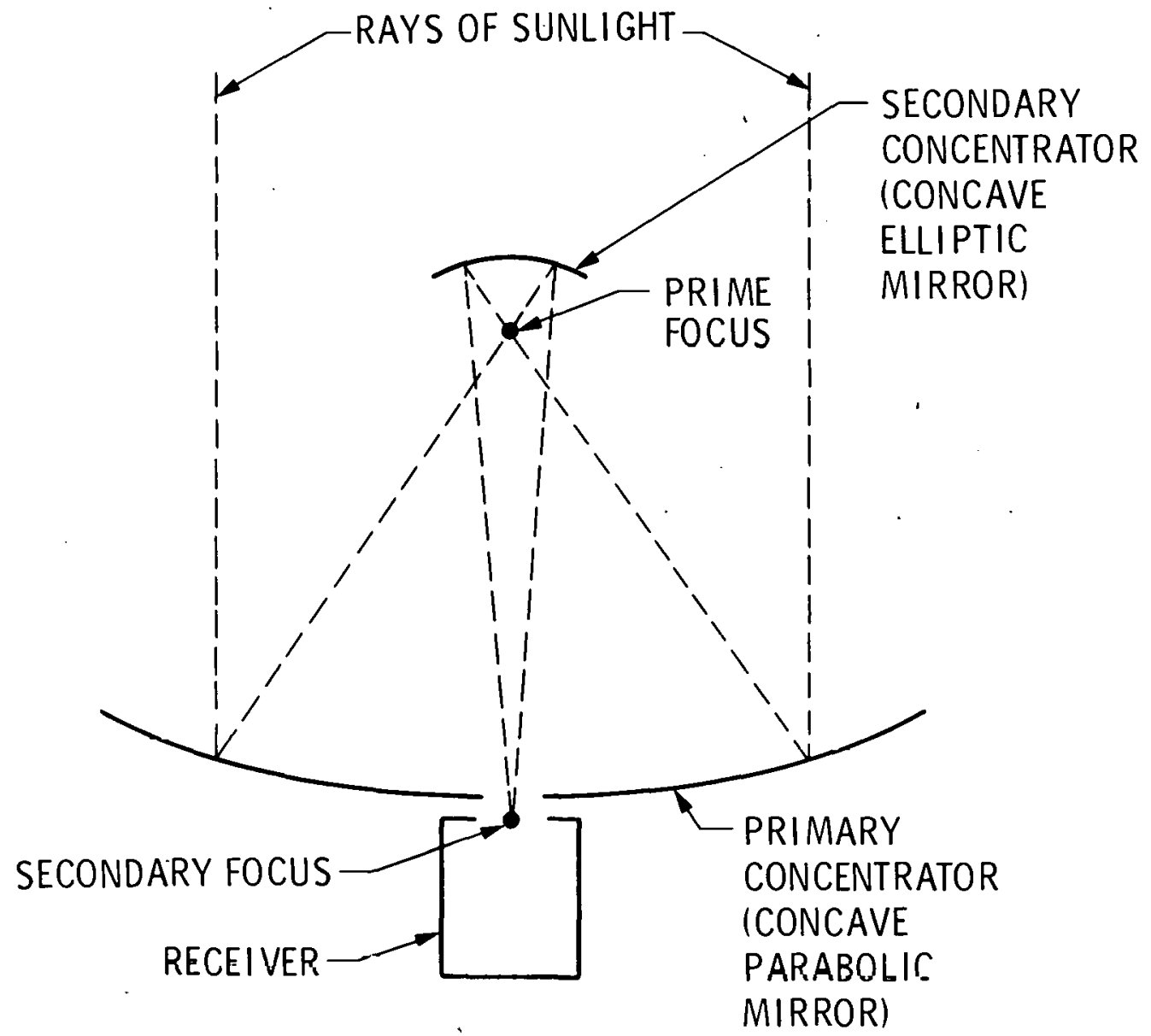

Figure 10. Folding Optical Path with a Secondary Mirror: Gregorian Configuration 
Coma and certain other optical aberrations can be corrected by using a primary that deviates appropriately from a parabolic shape, together with a suitable secondary. One such configuration is the Ritchey-Chrétien, a modified Cassegrainian in which the primary and secondary deviate slightly from parabolic and hyperbolic shapes, respectively. For a folded-beam concentrator, the Ritchey-Chrétien is preferable to a conventional Cassegrainian and should provide a significantly higher geometric concentration. The merits of this and other aberration-correcting configurations for solar concentrators should be investigated further.

\section{THREE-ELEMENT CONCENTRATORS}

A variety of three-element configurations may offer advantages for solar concentrators. For example, a Ritchey-Chrétien might be utilized to fold the beam, with a third element added close to the secondary focus to provide further correction of aberrations or to increase geometric concentration in other ways. This design combines the advantages of a folded optical path, a fairly short overall length, and a high geometric concentration ratio, at the expense of optical losses at three elements, which can be a severe penalty. A Cassegrainian concentrator with a CEC or CPC tertiary mirror (Figure 11) has been briefly examined (Refs. 18 and 33).

Another three-element configuration that may be worth evaluating uses a third mirror to fold the beam to a focus just beyond the secondary (Figure 12). This compound concentrator could have very short overall length. The three elements would permit a high degree of correction for aberrations, and thus could provide a high concentration ratio. The secondary would need a central hole to pass the beam from the tertiary mirror. 


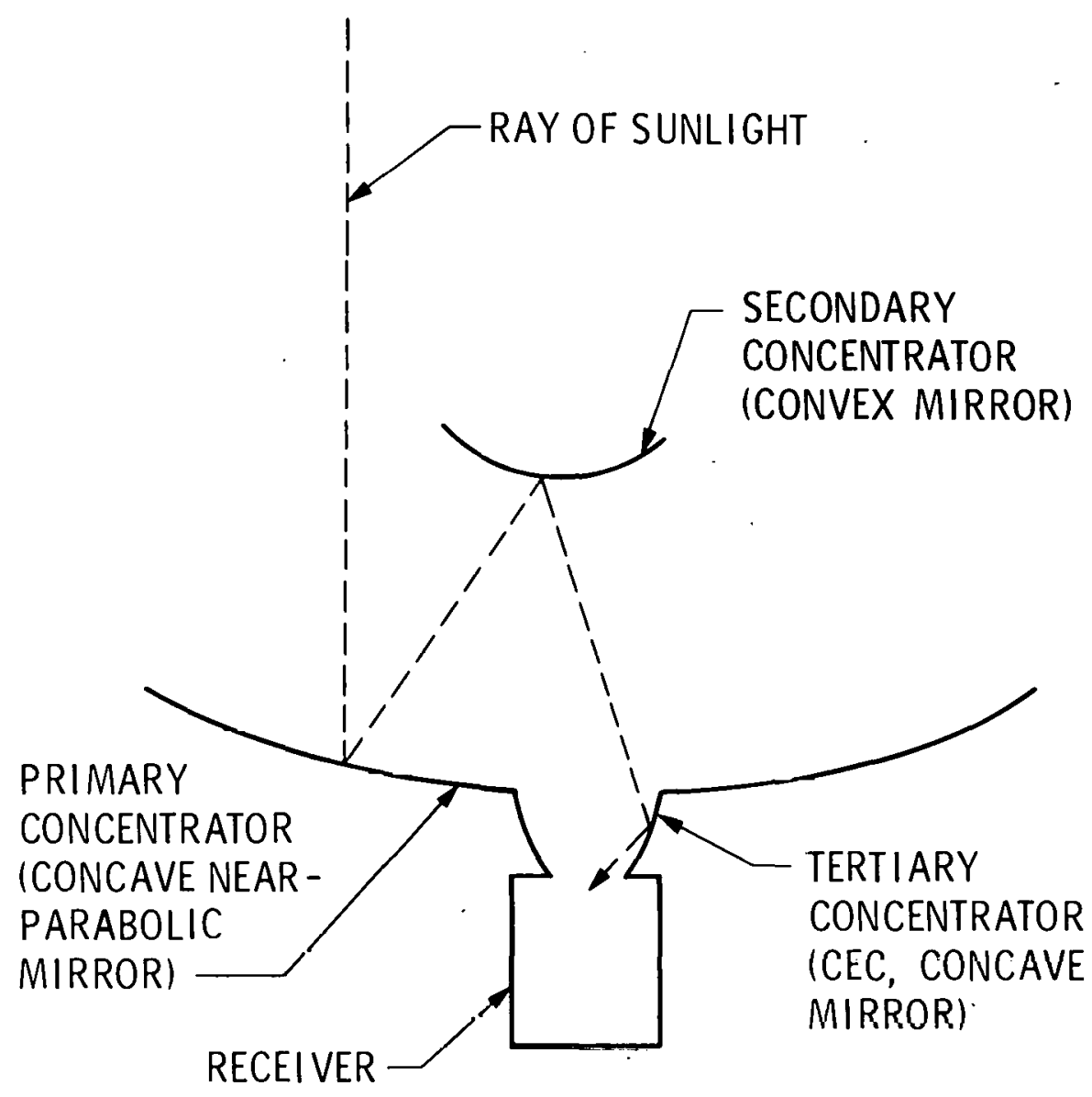

Figure 11. Folding Optical Path and Increasing Concentration Ratio Possible Three-Element Configuration: Cassegrainian or Ritchey-Chrétien plus CEC 


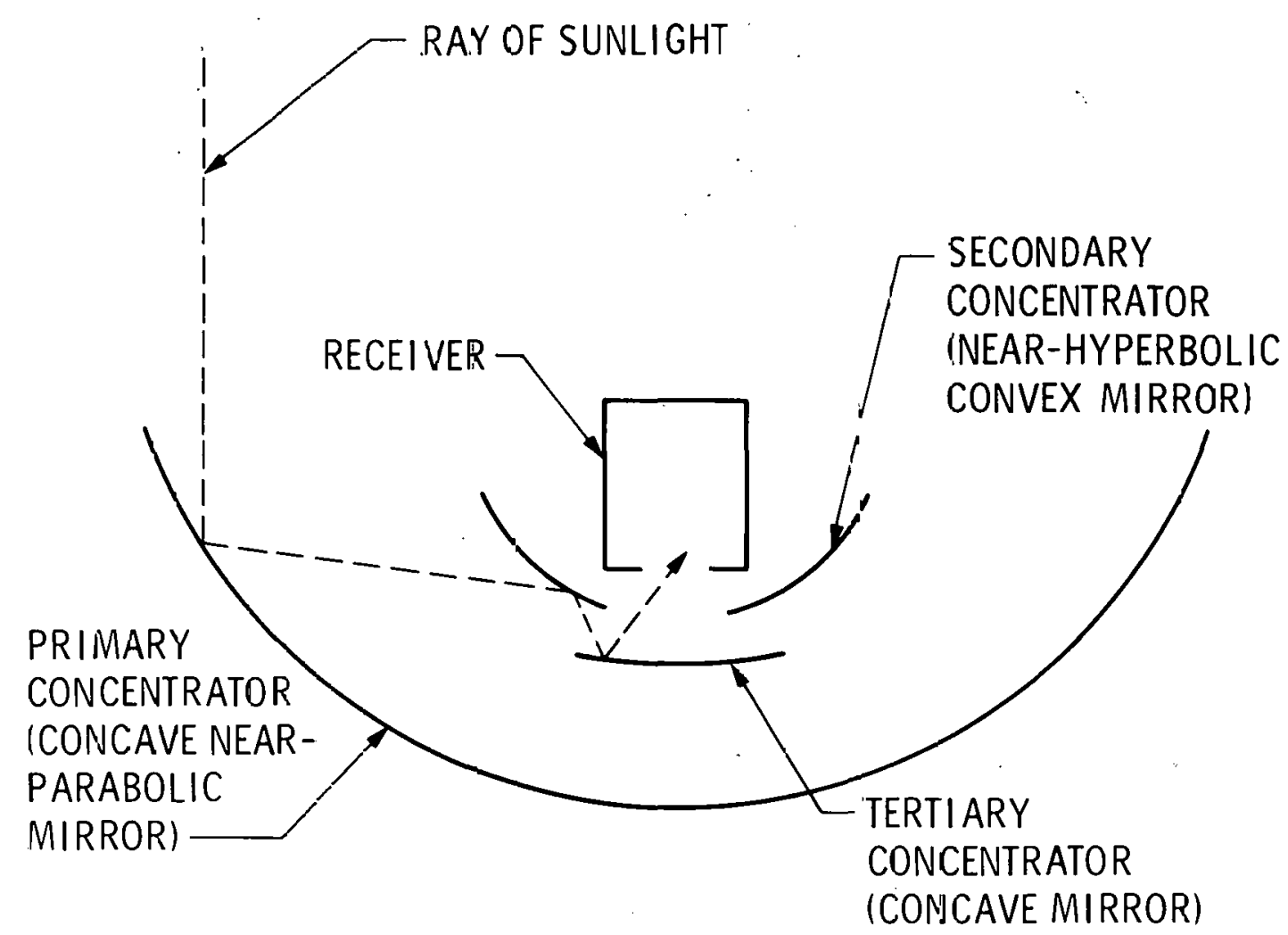

Figure 12. Folding Optical Path and Increasing Cancentration Ratio-Possible Three-Element Configuraょion: Short-Focus Ritchey-Chrétien plus Concave Correcting/Folding Mirror 


\section{SECTION $V$}

\section{AREAS NEEDING ATTENTION}

To permit adequate assessment of the advantages and disadvantages of the various optical geometries discussed above and their applicability to parabolic dish solar thermal power systems, work is needed in several technical areas. The se areas are considered briefly here.

\section{A. OPTICS}

Rather limited research has been done on the optical performance of the various compound configurations for solar thermal systems. It is not clear whether efficient optical analys is computer programs exist for many configurations. Such computer programs should preferably use cone optics (Refs. 34 through 39) rather than ray optics to reduce the computational cost and should take into account slope and position errors in the optics and lack of specularity. The output preferably should be in the form of a flux distribution at and near the focal plane and of intercept factor as a function of radial distance from the focal point in the focal plane. If lenses are used, thus introducing chromatic aberration, flux distribution should be integrated over the solar spectrum.

\section{B. HEAT TRANSFER}

As mentioned earlier, the heat flux incident upon a small secondary or tertiary will be fairly high. This is likely to cause problems with distortion, cracking, or oxidation. To dissipate the heat and to keep the secondary or tertiary temperature low, means for cooling must be provided. Depending upon the design and materials, these may be passive: radiation, free convection, and conduction; or active: forced convection of air, water, or other fluids. Apparently, no work in this area has been published for parabolic dish solar concentrators. Heat transfer calculations for representative designs are needed. Water-cooled laser optics are, however, currently available (Appendix B) and may provide a good starting point for solar work.

\section{MATERIALS}

For small secondaries or tertiaries, there is much incentive to use materials with high reflectance or transmittance to reduce optical losses in these elements and the associated heating problems. High reflectance may be difficult to maintain at high temperatures. Other material characteristics needed are minimum distortion, resistance to cracking and, depending on the design, good thermal conductivity.

For secondary and tertiary mirrors, a substrate of low-expansion or leached glass or one of metal with suitable high-temperature thermal, mechanical, and optical characteristics may be practical. A major problem may be finding a reflecting layer that will retain good reflectance at the temperature of service. Further effort is required in this area. One obvious approach is vapor-deposited aluminum protected from oxidation by a suitable inorganic coating. Water-cooled metal reflectors with front surfaces of aluminum, silver, and other materials and appropriate overcoats are being used successfully for laser optics (Appendix B). 
For secondary and tertiary lenses, heating probably will be too great for the lens to be made of plastic. Soda-lime glasses are inexpensive, but have high thermal expansion and tend to fracture under thermal shock. Tests to determine whether they can withstand the shock of sudden incidence of concentrated sunlight would be worthwhile. Low-expansion glasses such as borosilicate are probably preferable. Leached glass (Vycor) has lower thermal expansion, and fused silica still lower. Fused silica, however, cannot be molded and would have to be ground to shape, which would be expensive. Borosilicate and leached glasses can be molded and probably would be the most suitable materials. Molded glass Fresnel lenses are produced in quantity for marine use and for traffic signals. In mass production the cost should be only a few dollars per lens.

\section{SYSTEMS}

Compound concentrators must be evaluated as part of a solar thermal system. The pros and cons of increases in geometric concentration, changes in optical efficiency, changes in structural configuration, etc., depend upon consideration of the dish system as a whole.

One possibility that needs particular attention is the use of heat deposited in the secondary to preheat the working fluid going to the receiver. Whether this is practical or useful depends upon the power conversion cycle employed, the operating temperatures, etc. No analysis of this approach has been published to date. If the technique appears useful, it may be advantageous to integrate the secondary with the receiver as a terminal concentrator.

\section{E. PERTINENT WORK ON OTHER APPLICATIONS}

In addition to the limited effort underway in the various technical areas concerning compound concentrators for solar thermal use, there is also a body of work on similar problems for at least two other applications: solar thermophotovoltaics and laser optics. Such work is reviewed briefly in Appendixes $A$ and $B$. Some of this technology should be directly applicable to compound solar thermal concentrators, particularly to optics of compound concentrators, and in the design and fabrication of high-temperature optical elements using active cooling. 
For parabolic dish solar thermal power systems, compound concentrators offer possibilities of 1) folding the optical beam to reduce structural costs or to permit a better location for the receiver and power conversion equipment and of 2) increasing the geometric concentration ratio to reduce receiver losses or to maintain performance while relaxing tolerances or other requirements on the primary concentrator.

A wide variety of compound concentrators may be considered. Secondary elements of these concentrators may be lenses or mirrors, imaging or non-imaging.

A secondary concentrator does not necessarily lower optical efficiency. It may increase overall optical efficiency by increasing the intercept factor or by reducing optical aberrations.

A primary concentrator design which is optimum when used alone may not be optimum when used as part of a compound concentrator.

Further effort is required in the areas of optical analysis of compound concentrators, associated heat transfer, materials, system analysis, and design. Existing achievements in laser optics may prove applicable to the solar thermal field. 


\section{THIS PAGE}

\section{WAS INTENTIONALLY \\ LEFT BLANK}

32 


\section{REFERENCES}

1. L. D. Jaffe, "Dish Concentrators for Solar Thermal Energy: Status and Technology Development," DOE/JPL 1060-48, August 1981.

2. A. B. Meinel and M. P. Meinel, Applied Solar Energy, Addi son-Wesley, Reading, Massachusetts, 1976, pp. 150-15T, 164-165, 192-193, 227-228.

3. M. Collares-Pereira, A. Rabl, and R. Winston, "Lens-Mirror Combination wi th Maximal Concentration," Appl. Optics 16, pp. 2677-2683, 1977.

4. M. Collares-Pereira, J. O'Gallagher, A. Rabl, R. Winston, J. Egger, and K. Williams, "High Temperature Solar Collector of Optimal Concentration -. Non-Focusing Lens with Secondary Concentrator," Proc. International Solar Energy Society, New Dehli Meeting, pp. 1282-1286, January 1978.

5. A. Luque, "Quasi-optimum Pseudo-Lambertian Reflecting Concentrators: An Analysis," Appl. Optics 19, pp. 2398-2407, 1980.

6. Y. C. Wu and L: C. Wen, "Solar Receiver Performance of Point Focusing Collector Systems," ASME Reprint 78-WA/So 1-5, New York, 1978.

7. A. K. Alimov and D. N. Alavutidov, "Two-Mirror Unit with Plane and Hyperboloidal Counter-Reflectors," Geliotekhnika 15, No. 3, pp. 44-46, 1979.

8. Sundstrand Aviation-Denver, "Investigation of a 15-kW Solar Dynamic Power System For Space Application," Technical Report AFAPL-TR-64-156, Air Force Aero Propulsion Laboratory, Wright-Patterson Air Force Base, Ohio, February 28, 1965.

9. Sanders Associates, "Parametric Analysis Review, High Temperature Solar Thermal Receiver, JPL Contract \#955454," report to JPL, August 23, 1979.

10. W. T: Welford and R. Winston, Optics of Non-Imaging Concentrators, Academic Press, New York, New York, 1978.

11. V. K. Baranov, Optiko-Mekhanicheskaya Promyshlennost, No. 6, 1975.

12. V. K. Baranov, "Parabolntorndnidal Mirrors as. Elements of Solar Energy Concentrators," Geliotekhnika 2, No. 3, pp. 11-14, 1966.

13. R. Winston, "Principles of Solar Concentrators of a Novel Design," Solar Energy 16, pp. 89-95, 1974.

14. R. Winston, "Concentrating Collectors for Finite Sources," Applied Optics 17, pp. 688-689, 1978.

15. R. Winston and W. T. Welford, "Design of Non-Imaging Concentrators as Second Stages in Tandem with Image-Forming First Stage Concentrators," Applied Optics 19, pp. 347-351, 1980.

16. V. K. Baranov, "Parabolotoric Focone as Secondary Solar Energy Concentrator," Geliotekhnika 13, No. 5, pp. 18-25, 1977. 
17. P. Poon and S. Higgins, "Optical Performance of Several Point Focusing Solar Concentrators," presented at 86th National Meeting, Am. Inst. Chem. Engineers, Houston, Texas, April 1979.

18. W. T. Welford and R. Winston, "Design of Second-Stage Non-Imaging Concentrators," report to JPL, August 1979.

19. R. Winston and W. T. Welford, "The Geometric Vector Flux and Some New Non-Imaging Concentrators," J. Opt. Soc. Am. 69, pp. 532-535, 1979.

20. P. Greenman, J. O'Gallagher, R. Winston, and W. T. Welford, "Design of Non-Imaging Concentrators as Second Stages in Tandem wi th Image-Forming First Storage Concentrators," Proc. 1980 Annual Meeting, Am. Section, Int' 1 Solar Energy Soc., pp. 544-547, 1980.

21. R. Winston and W. T. Welford, private communication, 1980.

22. A. Meinel, private communication, 1980 .

23. E. I. Buzin, "Conical Concentrator with Point-Focusing Secondary," GeliotekhIIikd 4, No. 2, pp. 25-31, iyb8.

24. A. J. McDanal, "Optical Analysis of Paraboloidal Concentrators with Secondary Reflectors," presented at 1979 Annual Meeting, Int'1 Solar Energy Soc., Atlanta, Georgia, May 1979.

25. V. V. Novikov, V. K. Baranov, L. V. Vasilyeva, and N. N. Protosov, "Cassegrainian System Modified for Solar Concentrators," Geliotekhnika 2, No. 5, pp. 15-18, 1966.

26. G. Y. Umarov and D. N. Alavutdinov, "Investigation of Two-Mirror Systems Consisting of a Paraboloidal Mirror and an. Inflated-Film Reflector," Geliotekhnika 4, No. 3, pp. 62-66, 1968.

27. G. Y. Umarov, R. A. Zakhidov, and D. A. Kirqizbaev, "Determining the fienmetric Parameters of a Solar Power Element Consisting of a Hyperboloid and Paraboloid of Revolution," Geliotekhnika 9, No. 4, pp. 39-43, 1973.

28. D. A. Kirgizbaev and R. A. Zakhidov, "Energy Distribution in the Concentration Field of a Solar Installation with a Hyperboloidal Counter-Reflector," Geliotekhnika, No. 4, pp. 13-19, 1974.

29. R. A. Zakhidov and A. A. Vainer, "Integrated Precision Parameter for. a Cassegrainian System," Geliotekhnika 11. Nos, 3\&4, pp. 133-134, 1975.

30. G. Y. Umarov, A. K. Al imov, and D. N. Alavutdinov, "A Cassegrainian System for Solar Radiation," Geliotekhnika 12, No. 2, pp. 68-69, 1976.

31. R. A. Zakhidov and A. A. Vainer, "Paraboloid-Hyperboloid Concentrating Systems and Their Accuracy," Geliotekhnika 13, No. 1, pp. 42-49, 1977.

32. R. A. Zakhidov, "General Principles of Multielement Concentrating System Design," Geliotekhnika 14, No. 1, pp. 22-29, 1979.

33. M. H. Cobble, W. C. Hull, and R. A Hays, "Analysis of a Cassegrainian Solar 
Furnace," Society of Photo-Optical and Instrumentation Engineers, Conference Proceedings 161, pp. 55-63, 1978.

34. A. H. Bass, Jr., G. L. Schrenk, P. T. Y. Poon, and S. N. Higgins, "Optical Analysis of Cassegrainian Concentrator Systems," presented at 1979 Int'1 Congress, Int'1. Solar Energy Soc., Atlanta, Georgia, May 1979.

35. F. Cabannes and A. Le Phat Vinh, "Calcul de la repartition de 1 'energie solaire reflechie par un miroir parabolique," J. Physique Radium 15, 817 , 1954.

36. G. L. Schrenk, "Theoretical Analysis of Solar Reflectors," Allison Div., General Motors Corp., EDR 3139, July 1963.

37. G. L. Schrenk, "Analys is of Solar Reflectors -- Mathematical Theory and Methodology for Simulation of Real Reflectors," Final Report, Contract AF04 (695)-335, Allison Div., General Motors Corp., EDR 3693, December 1963.

38. P. T. Y. Poon and S. N. Higgins, "Optical Performance of a Fresnel-Type Concentrator wi th Truncated Paraboloidal Facets," presented at the Second International Helioscience Institute Conference on Alternate Energy Sources, Palm Springs, California, April 1978.

39. L. Wen, L. Huang, P. Poon, and W. Carley, "Comparative Study of Solar Optics for Paraboloidal Concentrators," Am. Soc. Mech. Eng. Preprint 79-WA/Sol-8, 1979.

40. W. Koechner, H. R. Verdun, N. C. Wyeth, R. E. Forkey, W. Davis, and R. Wientzen, "Technical Feasibility and Economic Assessment of Solar Thermophotovoltaic Conversion," presented at the EPRI Solar Energy Program Review, San Diego, California, September 1979.

41. D. R. 01 sen, J. R. Kurdock, and R. R. Rigby, "Fabrication Techniques for High Power Laser Optics, "Society of Photo-optical and Instrumentation Engineers 61, pp. 114-121, 1975.

42. Hughes Aircraft Company, "Smal1 Lightweight ALL Mirror Fabrication," Air Force Weapons Laboratory Technical Report 78-69, Kirtland Air Force Base, New Mexico, May 1979.

43. Pratt \& Whitney Aircraft Group, United Technologies, "Low Distortion Cooled Mirrors for High Power Lasers," West Palm Beach, Florida. 
THIS PAGE

\section{WAS INTENTIONALLY LEFT BLANK}


Work has been actively under way for several years on the feasibility of solar thermophotovoltaic conversion. This work has been sponsored primarily by the Electric Power Research Institute (EPRI).

The concept consists essentially of focusing sunlight into a cavity receiver whose wall constitutes a radiator. The exterior of this wall reradiates the absorbed energy into a second cavity, whose outer wall is composed of a photovoltaic cell. The unit is thus a photovoltaic device with two special features:

(1) The radiator acts as a frequency down-shifter, which converts the solar radiation to a spectral distribution better matched to the band-gap of silicon PV cells and hence increases their efficiency.

(2) The dual-cavity configuration greatly reduces losses from reflection and re-radiation.

Parametric study (Ref. 40) indicates that for efficient performance radiator temperatures of 1500 to $2000^{\circ} \mathrm{C}\left(2700\right.$ to $\left.3600^{\circ} \mathrm{F}\right)$ are needed, with geometric concentration ratios of 5,000 to 15,000. To achieve these concentrations, compound concentrators using a secondary concentration ratio of about 4 together with a primary concentration ratio of 2,500 to 3,500 have been examined, and the error tolerances analyzed. A design has been proposed which incorporates a faceted parabolic primary mirror and a CPC secondary. A window over the secondary entrance aperture would be used to further reduce thermal losses; both the secondary mirror and the photovoltaic cells would be water-cooled (Ref. 40). This work may be useful in the design of compound optics for solar thermal power systems. 
THIS PAGE

\section{WAS INTENTIONALLY \\ LEFT BLANK}




\section{APPENDIX B}

\section{CURRENT WORK ON LASER OPTICS}

Interest in high-energy lasers has led to development effort on laser optics. The radiant fluxes which must be handled range from 5,000 to $1,000,000 \mathrm{~kW} / \mathrm{m}^{2}$.

Water-cooled mirrors have been developed for such applications. Typically, they utilize a substrate of copper or molybdenum upon which is deposited a reflecting layer of silver or a dielectric coating tuned to provide high reflectivity at the laser wavelength. An evaporated overcoating, such as thorium fluoride, may be applied over the silver to retard tarnishing. The substrate is brazed to a heat exchanger of the same material which defines the channels through which the cooling water flows. Optics of this kind are commercially available. More information is given in References 41 through 43 .

This work should be of help in developing water-cooled secondary mirrors if they are needed for solar thermal power systems. 